\title{
DISTANCES BETWEEN BANACH SPACES
}

\author{
N.J.Kalton And M.I.OstrovskiI
}

\begin{abstract}
The main object of the paper is to study the distance between Banach spaces introduced by Kadets. For Banach spaces $X$ and $Y$, the Kadets distance is defined to be the infimum of the Hausdorff distance $d\left(B_{X}, B_{Y}\right)$ between the respective closed unit balls over all isometric linear embeddings of $X$ and $Y$ into a common Banach space $Z$. This is compared with the Gromov-Hausdorff distance which is defined to be the infimum of $d\left(B_{X}, B_{Y}\right)$ over all isometric embeddings into a common metric space $Z$. We prove continuity type results for the Kadets distance including a result that shows that this notion of distance has applications to the theory of complex interpolation.
\end{abstract}

\section{INTRODUCTION}

The standard notion of distance between two Banach spaces is the Banach-Mazur distance which is defined by

$$
d_{B M}(X, Y)=\log \inf \left\{\|T\|\left\|T^{-1}\right\|: T: X \rightarrow Y \text { is an isomorphism }\right\} .
$$

(It is usual to omit the logarithm, but for consistency we will include it). The Banach-Mazur distance is only finite when $X$ and $Y$ are isomorphic. The main object of this paper is to study a measure of distance we call the Kadets distance and certain related notions of distance. The Kadets distance has natural applications in interpolation theory which we explain.

We recall that if $Z$ is a Banach space and $X$ and $Y$ are closed subspaces of $Z$ the gap or opening $\Lambda(X, Y)$ is defined as the Hausdorff distance between the closed unit balls $B_{X}$ and $B_{Y}$ of $X$ and $Y$ i.e.

$$
\Lambda(X, Y)=\max \left\{\sup _{y \in B_{Y}} d\left(y, B_{X}\right), \sup _{x \in B_{X}} d\left(x, B_{Y}\right)\right\} .
$$

If $X$ and $Y$ are arbitrary Banach spaces we define the Kadets distance

$$
d_{K}(X, Y)=\inf _{Z, U, V} \Lambda(U X, V Y)
$$

where the infimum is taken over all Banach spaces $Z$ and all linear isometric embeddings $U: X \rightarrow Z$ and $V: Y \rightarrow Z$.

This distance was apparently introduced by Kadets [15] who proved for example that $\lim _{p \rightarrow 2} d_{K}\left(\ell_{p}, \ell_{2}\right)=0$. However the basic idea seems to be implicit in some

1991 Mathematics Subject Classification. Primary 46B20, 46M35, Secondary 46B03, 54E35.

Key words and phrases. Banach Space, Opening between Subspaces, Gap between Subspaces, Complex Interpolation Family, Gromov-Hausdorff Distance, Quasi-Banach Space. 
earlier work of Krein, Krasnoselskii and Milman [20], Brown [5] and Douady [10]. The second author studied the notion in [22] and proved that $d_{K}$ satisfies the triangle law but that there are non-isomorphic Banach spaces $X$ and $Y$ for which $d_{K}(X, Y)=0$ (thus $d_{K}$ is a "pseudo-metric"). In the same paper there is a completeness result: if $\left(X_{n}\right)$ is a sequence of Banach spaces Cauchy with respect to $d_{K}$ then there is a Banach space $X$ so that $\lim _{n \rightarrow \infty} d_{K}\left(X_{n}, X\right)=0$.

There is a series of papers studying the general problem of the identifying properties which are stable under small perturbations in the Kadets distance (see [2], [5] [10], [20], [22], [23], [24] and [25]). Precisely a property $\mathcal{P}$ is called stable if there exists $\epsilon>0$ so that if $X$ has $\mathcal{P}$ and $d_{K}(X, Y)<\epsilon$ then $Y$ has $\mathcal{P}$. We refer to the survey article [24] Chapter 6: a partial list of stable properties includes reflexivity, super-reflexivity, B-convexity (nontrivial Rademacher type), the Banach-Saks property, the alternate-signs Banach Saks property and the property of not containing $\ell_{1}$.

The Kadets distance is clearly related to the notion of Gromov-Hausdorff distance between metric spaces (see [11],[27]; the precise definition is given in Section 2 ). It is natural to introduce the Gromov-Hausdorff distance between two Banach spaces $X$ and $Y$ as

$$
d_{G H}(X, Y)=\inf _{Z, U, V} d\left(U B_{X}, V B_{Y}\right)
$$

where the infimum is taken over all isometries of $U: X \rightarrow Z$ and $V: Y \rightarrow Z$ into a common metric space $Z$ (here $d\left(U B_{X}, V B_{Y}\right)$ is the Hausdorff distance between $U B_{X}$ and $V B_{Y}$.) Thus the Gromov-Hausdorff distance is simply the nonlinear analogue of the Kadets distance. It is not difficult to see that this definition coincides with computing the standard Gromov-Hausdorff distance between the unit balls $B_{X}, B_{Y}$ as metric spaces. Let us remark at this point that a related global notion was considered in [4] and [12].

In this paper we first compare these two notions of distance. It is worth pointing out that one must distinguish between the case of complex scalars and real scalars, because there are examples $([3],[17],[31])$ of complex Banach spaces which are realisometric but not even complex-isomorphic. Gromov-Hausdorff distance cannot distinguish complex structures.

We show that (for real scalars) while Gromov-Hausdorff distance is not equivalent to the Kadets distance, the two notions are equivalent if one restricts to Banach spaces which are nice enough. For example if $X$ is B-convex (i.e. has non-trivial Rademacher type) or if $X^{*}$ embeds into an $\mathcal{L}_{1}$-space then $d_{G H}\left(X_{n}, X\right) \rightarrow 0$ implies $d_{K}\left(X_{n}, X\right) \rightarrow 0$. If $X$ is isomorphic to either $c_{0}$ or $\ell_{\infty}$ then $d_{G H}\left(X_{n}, X\right) \rightarrow 0$ implies $d_{B M}\left(X_{n}, X\right) \rightarrow 0$. On the other hand $d_{G H}\left(\ell_{p}, \ell_{1}\right) \rightarrow 0$ as $p \rightarrow 1$ while $d_{K}\left(\ell_{p}, \ell_{1}\right)=1$ if $p>1$. The precise identification of the class on which the two distances are equivalent is related to the notion of a $\mathcal{K}$-space introduced in [16] (see [18]) and to the theory of twisted sums.

In fact for real scalars, Gromov-Hausdorff distance is equivalent to a notion of distance analogous to the Kadets distance but allowing the superspace $Z$ to be a quasi-Banach space.

These results are developed in Section 3, after some preliminary results in Section 2. In Section 4, we then apply our techniques to prove a number of continuity-type results for the Kadets metric. For example we show that in an obvious sense the map $X \rightarrow X^{*}$ is continuous for the Kadets metric, and even more one has 
$\left(X_{0}, X_{1}\right)$ is a complex Banach couple and $X_{\theta}=\left[X_{0}, X_{1}\right]_{\theta}$ are the spaces obtained by the (Calderoń) method of complex interpolation (cf. [6]) then the map $\theta \rightarrow X_{\theta}$ is continuous for the Kadets distance for $0<\theta<1$. This result is closely related to recent work on uniform homeomorphisms between the unit balls of two Banach spaces using complex interpolation methods (cf. [8]). We give precise estimates here and obtain the estimate for $1<p, q<\infty$,

$$
d_{K}\left(\ell_{p}, \ell_{q}\right) \leq 2 \frac{\sin (\pi|1 / p-1 / q| / 2)}{\sin (\pi(1 / p+1 / q) / 2)}
$$

which improves earlier estimates (see [15], [22]). We remark that in [23] or [24] (pp. $292,303)$ there is a lower estimate $d_{K}\left(\ell_{p}, \ell_{q}\right) \geq \frac{1}{2}\left(2^{1 / p}-2^{1 / q}\right)$.

Finally in Section 5, we make some remarks on the topology of the pseudometric space of all Banach spaces with a given density character with either notion of distance. We point out that results on stability or openness of some property lead automatically to results on complex interpolation spaces, and also show that the continuity results of the previous section lead to new stable or open properties. We identify the component of $\ell_{1}$ for the Kadets distance and raise the question of identifying the components of $\ell_{2}$ and $c_{0}$. We do not know if the set of separable Banach spaces is connected for the Gromov-Hausdorff distance. We also show that if $1<p \neq 2<\infty$ the set of spaces isomorphic to $\ell_{p}$ is non-separable for both notions of distance.

\section{Gromov-Hausdorff distance and Kadets distance.}

We first recall the notion of Gromov-Hausdorff distance between metric spaces. It will be convenient to expand the definition to include pseudo-metric spaces. We recall that if $M$ is a set, a pseudo-metric on $M$ is a map $d: M \times M \rightarrow[0, \infty)$ which is symmetric and satisfies the triangle law, the condition $d(x, x)=0$, but not necessarily the condition $d(x, y)=0$ implies $x=y$. Suppose $A$ and $B$ are metric spaces (or pseudo-metric spaces) with bounded metrics. We define the GromovHausdorff distance between $A$ and $B$ denoted $d_{g h}(A, B)$ to be the infimum of all $\epsilon \geq 0$ so that there is a pseudo-metric space $M$ and isometric embeddings $i_{A}$ : $A \rightarrow M$ and $i_{B}: B \rightarrow M$ such that the Hausdorff distance $d_{M}\left(i_{A} A, i_{B} B\right) \leq \epsilon$. If $\mathcal{M}$ is any set of metric spaces then $d_{g h}$ defines a pseudo-metric on $\mathcal{M}$; note that $d_{g h}(A, B)=0$ does not necessarily imply that $A$ and $B$ are isometric (unless they are compact).

We will be interested in an alternative formulation. For convenience we denote by $\mathcal{F}(A, B)$ the collection of all pairs $(\phi, \psi)$ of maps $\phi: A \rightarrow B$ and $\psi: B \rightarrow A$. If $(\psi, \phi)$ is such pair let $G=G(\phi, \psi)$ be the union of the graphs of $\psi, \phi$. We define $D(\phi, \psi)$ to be the supremum of all quantities $\frac{1}{2}\left|d_{A}\left(a_{1}, a_{2}\right)-d_{B}\left(b_{1}, b_{2}\right)\right|$ over all $a_{1}, a_{2} \in A, b_{1}, b_{2} \in B$ with $\left(a_{i}, b_{i}\right) \in G$ for $i=1,2$.

In the special case when $\phi$ is invertible and $\psi=\phi^{-1}, D(\phi, \psi)$ is the supremum of all quantities $\frac{1}{2}\left|d_{B}\left(\phi a_{1}, \phi a_{2}\right)-d_{A}\left(a_{1}, a_{2}\right)\right|$ where $a_{1}, a_{2} \in A$.

Theorem 2.1. Let $A$ and $B$ be bounded metric spaces. Then

$$
d_{g h}(A, B)=\inf _{(\phi, \psi) \in \mathcal{F}} D(\phi, \psi) .
$$

Proof. First suppose $M$ is a pseudo-metric space and that $A$ and $B$ are isometrically 
can define $\phi: A \rightarrow B$ and $\psi: B \rightarrow A$ so that $d_{M}(a, \phi a), d_{M}(\psi b, b)<\sigma$ for all $a \in A$ and $b \in B$. Now for $\left(a_{i}, b_{i}\right) \in G=G(\phi, \psi)$ we clearly have $\left|d_{A}\left(a_{1}, a_{2}\right)-d_{B}\left(b_{1}, b_{2}\right)\right|<$ $2 \sigma$.

To obtain the converse direction suppose $(\phi, \psi)$ given and that $D(\psi, \phi)=\sigma$. We let $M=A \cup B$ (disjoint union) and define a pseudo-metric $d_{M}$ as follows. We let $d_{M}$ coincide with $d_{A}$ on $A$ and with $d_{B}$ on $B$. If $a \in A$ and $b \in B$ then

$$
d_{M}(a, b)=\inf _{\left(a^{\prime}, b^{\prime}\right) \in G}\left(d_{A}\left(a, a^{\prime}\right)+d_{B}\left(b^{\prime}, b\right)\right)+\sigma .
$$

We must check this is a pseudo-metric on $A \cup B a_{1}, a_{2} \in A$ and $b \in B$. We check that $d_{A}\left(a_{1}, a_{2}\right) \leq d_{M}\left(a_{1}, b\right)+d_{M}\left(a_{2}, b\right)$. To do this suppose that $\left(\alpha_{1}, \beta_{1}\right)$ and $\left(\alpha_{2}, \beta_{2}\right) \in G$. Then

$$
\begin{aligned}
d_{B}\left(\beta_{1}, b\right)+d_{B}\left(\beta_{2}, b\right) & \geq d_{B}\left(\beta_{1}, \beta_{2}\right) \\
& \geq d_{A}\left(\alpha_{1}, \alpha_{2}\right)-2 \sigma .
\end{aligned}
$$

Hence

$$
d_{A}\left(a_{1}, \alpha_{1}\right)+d_{B}\left(\beta_{1}, b\right)+d_{A}\left(\alpha_{2}, a_{2}\right)+d_{B}\left(\beta_{2}, b\right) \geq d_{A}\left(a_{1}, a_{2}\right)-2 \sigma
$$

which establishes our claim.

We can also show that $d_{M}\left(a_{1}, b\right) \leq d_{A}\left(a_{1}, a_{2}\right)+d_{M}\left(a_{2}, b\right)$. We omit the details which are easy. Arguing symmetrically with $A, B$ interchanged gives the conclusion that $d_{M}$ is a pseudo-metric. Clearly $d_{M}(a, \phi a) \leq \sigma$ and $d_{M}(\psi b, b) \leq \sigma$. This shows that $d_{g h}(A, B) \leq \sigma$.

If $X$ and $Y$ are Banach spaces we define the Gromov-Hausdorff distance $d_{G H}(X, Y)$ to be the Gromov-Hausdorff distance between their closed unit balls $B_{X}, B_{Y}$, i.e. $d_{G H}(X, Y)=d_{g h}\left(B_{X}, B_{Y}\right)$. Equivalently $d_{G H}(X, Y)$ is the infimum of the Hausdorff distance $d\left(B_{X}, B_{Y}\right)$ over all isometric embeddings of $X, Y$ into a common metric space $M$. To establish this last comment, suppose $d$ is any metric on the formal union $B_{X} \cup B_{Y}$, which coincides with the respective norm-distances on $B_{X}$ and $B_{Y}$. We can extend $d$ to $X \cup Y$ by defining $d$ again to coincide with the norm-distance on each of $X$ and $Y$ and for $x \in X, y \in Y$,

$$
d(x, y)=\inf _{u \in B_{X}, v \in B_{Y}}\left\{\|x-u\|_{X}+d(u, v)+\|y-v\|_{Y}\right\}
$$

We leave the details to the reader.

Let us note here that our definition applies to both real and complex Banach spaces, but there are complex Banach spaces which are real-isometric and not even complex-isomorphic ([3], [17], [31]). In view of this, Gromov-Hausdorff distance is most natural for the category of real Banach spaces.

Corollary 2.2. If $X$ and $Y$ are Banach spaces and $d_{G H}(X, Y)<\sigma$ then there exist maps $\phi: B_{X} \rightarrow B_{Y}$ and $\psi: B_{Y} \rightarrow B_{X}$ such that:

$$
\left|\|x-\psi(y)\|_{X}-\|y-\phi(x)\|_{Y}\right|<2 \sigma
$$

whenever $x \in B_{X}, y \in B_{Y}$.

The Kadets distance $d_{K}(X, Y)$ is defined to be the infimum of the gap $\Lambda(X, Y)(=$ 
space $Z$. Here our definition can equally be applied to the real or complex case. The Kadets distance is again a pseudo-metric on any set of Banach spaces (see [15] [22]). We clearly have the inequality $d_{G H}(X, Y) \leq d_{K}(X, Y)$.

We now give a similar formulation of the Kadets distance. Let $\mathcal{F}_{h}(X, Y)$ be the set of all pairs of homogenous maps $\Phi: X \rightarrow Y$ and $\Psi: Y \rightarrow X$ such that $\|\Phi(x)\|_{Y} \leq\|x\|_{X}$ and $\|\Psi(y)\|_{X} \leq\|y\|_{Y}$ for all $x \in X$ and $y \in Y$. We define $\Delta=\Delta(\Phi, \Psi)$ to be the least constant such that

$$
\left|\left\|\sum_{i=1}^{m} x_{i}-\sum_{j=1}^{n} \Psi\left(y_{j}\right)\right\|_{X}-\left\|\sum_{i=1}^{m} \Phi\left(x_{i}\right)-\sum_{j=1}^{n} y_{j}\right\|_{Y}\right| \leq \Delta\left(\sum_{i=1}^{m}\left\|x_{i}\right\|_{X}+\sum_{j=1}^{n}\left\|y_{j}\right\|_{Y}\right)
$$

for all $x_{1}, \ldots, x_{m} \in X$ and $y_{1}, \ldots, y_{n} \in Y$.

In the special case when $\Psi=\Phi^{-1}$ (and hence $\Phi$ is norm-preserving) notice $\Delta$ is the least constant such that

$$
\left|\left\|\sum_{i=1}^{m} \Phi\left(x_{i}\right)\right\|_{Y}-\left\|\sum_{i=1}^{m} x_{i}\right\|_{X}\right| \leq \Delta \sum_{i=1}^{m}\left\|x_{i}\right\|_{X}
$$

Theorem 2.3. If $X, Y$ are Banach spaces then

$$
d_{K}(X, Y)=\inf _{(\Phi, \Psi) \in \mathcal{F}_{h}} \Delta(\Psi, \Phi)
$$

Proof. If $X$ and $Y$ are isometrically embedded in $Z$ and $d\left(B_{X}, B_{Y}\right)<\sigma$ we can construct $(\Phi, \Psi) \in \mathcal{F}_{h}$ with $\|x-\Phi(x)\|_{Z} \leq \sigma\|x\|_{X}$ and $\|y-\Psi(y)\|_{Y} \leq \sigma\|y\|_{Y}$ whenever $x \in X$ and $y \in Y$. It is then trivial that $\Delta(\Phi, \Psi) \leq \sigma$.

For the converse direction, let us suppose that $(\Phi, \Psi) \in \mathcal{F}_{h}(X, Y)$ are given and that $\Delta(\Phi, \Psi)=\sigma$. We will define $Z$ to be the direct sum $X \oplus Y$ equipped with an equivalent norm. Precisely we define

$$
\|(u, v)\|_{Z}=\inf \left\{\left\|x_{0}\right\|_{X}+\left\|y_{0}\right\|_{Y}+\sigma\left(\sum_{i=1}^{m}\left\|x_{i}\right\|_{X}+\sum_{j=1}^{n}\left\|y_{j}\right\|_{Y}\right)\right\}
$$

where the infimum is taken over all $\left\{x_{i}\right\}_{i=0}^{m}$ in $X$ and $\left\{y_{j}\right\}_{j=0}^{n}$ in $Y$ such that

$$
u=x_{0}+\sum_{i=1}^{m} x_{i}+\sum_{j=1}^{n} \Psi\left(y_{j}\right)
$$

and

$$
v=y_{0}+\sum_{i=1}^{m} \Phi\left(x_{i}\right)+\sum_{j=1}^{n} y_{j}
$$

We show that $\|(u, 0)\|_{Z}=\|u\|_{X}$ (and then it follows similarly that $\|(0, v)\|_{Z}=$ $\left.\|v\|_{Y .}\right)$ Indeed suppose

$$
u=x_{0}+\sum^{m} x_{i}+\sum^{n} \Psi\left(y_{j}\right)
$$


and

$$
0=y_{0}+\sum_{i=1}^{m} \Phi\left(x_{i}\right)+\sum_{j=1}^{n} y_{j}
$$

It follows from our condition that

$$
\left\|\sum_{i=1}^{m} x_{i}+\sum_{j=1}^{n} \Psi\left(y_{j}\right)\right\|_{X} \leq\left\|y_{0}\right\|_{Y}+\sigma\left(\sum_{i=1}^{m}\left\|x_{i}\right\|_{X}+\sum_{j=1}^{n}\left\|y_{j}\right\|_{Y}\right) .
$$

Hence

$$
\|u\|_{X} \leq\left\|x_{0}\right\|_{X}+\left\|y_{0}\right\|_{Y}+\sigma\left(\sum_{i=1}^{m}\left\|x_{i}\right\|_{X}+\sum_{j=1}^{n}\left\|y_{j}\right\|_{Y}\right) .
$$

This shows that $\|(u, 0)\|_{Z}=\|u\|_{X}$.

Clearly the construction yields that $\Lambda(X, Y) \leq \sigma$ since $\|(x, \Phi(x))\|_{Z} \leq \sigma\|x\|_{X}$ and $\|(\Psi(y), y)\|_{Z} \leq \sigma\|y\|_{Y}$.

There is an amusing way to interpret $\Delta(\Phi, \Psi)$. Form the vector sequence spaces $\ell_{1}(X)$ and $\ell_{1}(Y)$ and define $\tilde{\Phi}\left(\left(x_{n}\right)\right)=\left(\Phi\left(x_{n}\right)\right)$ and $\tilde{\Psi}\left(\left(y_{n}\right)\right)=\left(\Psi\left(y_{n}\right)\right)$. We can consider the pair $(\tilde{\Phi}, \tilde{\Psi}) \in \mathcal{F}_{h}\left(\ell_{1}(X), \ell_{1}(Y)\right)$. Then consider the unit ball $B_{\ell_{1}(X)}$ with the pseudo-metric induced by the seminorm $\left|\left(x_{n}\right)\right|_{X}=\left\|\sum_{n=1}^{\infty} x_{n}\right\|_{X}$; similarly consider $B_{\ell_{1}(Y)}$ with the seminorm $\left|\left(y_{n}\right)\right|_{Y}$ defined in the analogous way.

Then $\Delta(\Phi, \Psi)=D(\tilde{\Phi}, \tilde{\Psi})$ for the unit balls of $\ell_{1}(X)$ and $\ell_{1}(Y)$ equipped with the pseudo-metrics induced by these seminorms. We leave the details to the reader.

It is natural to ask for a theorem of the type of Theorem 2.3 but with $\Phi$ a bijection and $\Psi=\Phi^{-1}$. This can be done at the cost of a constant in the calculations.

Theorem 2.4. Let $X$ and $Y$ be a Banach spaces with $d_{K}(X, Y)<\sigma<\frac{1}{6}$. Then there is a norm-preserving bijection $\Omega: X \rightarrow Y$ such that if $x_{1}, \ldots, x_{n} \in X$ then

$$
\left|\left\|\sum_{i=1}^{n} \Omega\left(x_{i}\right)\right\|_{Y}-\left\|\sum_{i=1}^{n} x_{i}\right\|_{X}\right| \leq 14 \sigma \sum_{i=1}^{n}\left\|x_{i}\right\|_{X}
$$

Proof. First we observe that if $d_{K}(X, Y)<1 / 2$ then $X$ and $Y$ have the same density character (cf. [20] or [24] 6.23). We will suppose that $X$ and $Y$ are simultaneously embedded into a common Banach space $Z$ with $d\left(B_{X}, B_{Y}\right)=\delta<\sigma$. Let us pick a maximal collection of vectors $\left(x_{i}: i \in I\right)$ in $S_{X}$ such that if $|a|=1$ and $i \neq j$ then $\left\|x_{i}-a x_{j}\right\|_{X}>4 \sigma$. Then there exist vectors $\left(y_{i}: i \in I\right)$ in $S_{Y}$ with $\left\|x_{i}-y_{i}\right\|_{Z}<2 \delta$. If $y \in S_{Y}$ then there exists $x \in S_{X}$ with $\|y-x\|_{Z}<2 \delta$ and so there exists $i \in I$ and $|a|=1$ such that $\left\|y-a y_{i}\right\|_{Z}<8 \sigma$. On the other hand if $i \neq j$ and $|a|=1$ then $\left\|y_{i}-a y_{j}\right\|_{Z}>4(\sigma-\delta)$.

We can now partition $S_{X}$ into sets $\left(A_{i}: i \in I\right)$ such that if $x \in A_{i}$ and $|a|=1$ then $a x \in A_{i}$ and further that $\left\|x-x_{i}\right\|_{X} \leq 2 \sigma$ implies $x \in A_{i}$ while $x \in A_{i}$ implies that there exists $a$ with $|a|=1$ and $\left\|x-a x_{i}\right\|_{X} \leq 4 \sigma$. We then define $A_{i}^{\prime}$ by taking one representative $u$ from each set $\{a x:|a|=1\}$ contained in $A_{i}$ with the property $\left\|u-x_{i}\right\|_{X} \leq 4 \sigma$.

In the same way we can partition $S_{Y}$ into sets $\left(B_{i}: i \in I\right)$ such that if $y \in B_{i}$ and $|a|=1$ then $a y \in B_{i}$ and further that $\left\|y-y_{i}\right\|_{X} \leq 2(\sigma-\delta)$ implies $y \in B_{i}$ 
define $B_{i}^{\prime}$ by taking one representative $v$ from each set $\{a y:|a|=1\}$ contained in $B_{i}$ with the property $\left\|v-y_{i}\right\|_{X} \leq 8 \sigma$.

It is easy to see that the sets $A_{i}^{\prime}, B_{i}^{\prime}$ have the same cardinality for each $i$ and so we can define a bijection $\Omega: S_{X} \rightarrow S_{Y}$ such that $\Omega\left(A_{i}^{\prime}\right)=B_{i}^{\prime}, \Omega(a x)=a \Omega(x)$ when $|a|=1$. If $x \in S_{X}$ then there exists $i \in I$ and $|a|=1$ with ax $\in A_{i}^{\prime}$. Thus $\Omega(a x) \in B_{i}^{\prime}$ and so $\|x-\Omega(x)\|_{Z} \leq 14 \sigma$.

The result now follows immediately.

Let us give one immediate application of Theorem 2.3.

Theorem 2.5. Let $X$ and $Y$ be real Banach spaces and suppose $Z$ is a metric linear space equipped with an invariant metric $d_{Z}$. Suppose $X$ and $Y$ are linearly and isometrically embedded into $Z$. Then $d_{K}(X, Y) \leq d_{Z}\left(B_{X}, B_{Y}\right)$.

Remark. Thus it would make no difference in the definition of $d_{K}$ to allow $Z$ to be a metric linear space instead of a Banach space.

Remark. The proof given below can be extended to complex spaces, if we further assume that the metric $d_{Z}$ is invariant under multiplication by $e^{i \theta}$ for $0<\theta<$ $2 \pi$. For the real case invariance under multiplication by -1 follows from additive invariance since $d_{Z}(-x,-y)=d_{Z}(x-y, 0)=d_{Z}(x, y)$.

Proof. Suppose $\sigma>d_{Z}\left(B_{X}, B_{Y}\right)$. Then we can define $\Phi: S_{X} \rightarrow B_{Y}$ and $\Psi$ : $S_{Y} \rightarrow B_{X}$ such that $d_{Z}(x, \Phi(x)) \leq \sigma$ and $d_{Z}(\Psi(y), y) \leq \sigma$ for all $x, y$. Since the metric is translation-invariant it is clear that we can suppose $\Phi(-x)=-\Phi(x)$ and $\Phi(-y)=\Phi(y)$. We then extend $\Phi$ and $\Psi$ to be homogenous.

Now if $x_{1}, \ldots, x_{m} \in S_{X}$ and $y_{1}, \ldots, y_{n} \in S_{Y}$ then

$$
d_{Z}\left(\sum_{i=1}^{m} x_{i}+\sum_{j=1}^{n} \Psi\left(y_{j}\right), \sum_{i=1}^{m} \Phi\left(x_{i}\right)+\sum_{j=1}^{n} y_{j}\right) \leq(m+n) \sigma
$$

It follows that since $\|x\|_{X}=d_{Z}(x, 0)$ for $x \in X$ and $\|y\|_{Y}=d_{Z}(y, 0)$ for $y \in Y$ we have:

$$
\left|\left\|\sum_{i=1}^{m} x_{i}+\sum_{j=1}^{n} \Psi\left(y_{j}\right)\right\|_{X}-\left\|\sum_{i=1}^{n} \Phi\left(x_{i}\right)+\sum_{j=1}^{n} y_{j}\right\|_{Y}\right| \leq(m+n) \sigma .
$$

From this it follows easily that if $r_{1}, \ldots, r_{m}, s_{1}, \ldots, s_{n}$ are integers that

$$
\left|\left\|\sum_{i=1}^{m} r_{i} x_{i}+\sum_{j=1}^{n} \Psi\left(s_{j} y_{j}\right)\right\|_{X}-\left\|\sum_{i=1}^{n} \Phi\left(r_{i} x_{i}\right)+\sum_{j=1}^{n} s_{j} y_{j}\right\|_{Y}\right| \leq\left(\sum_{i=1}^{m} r_{i}+\sum_{j=1}^{n} s_{j}\right) \sigma .
$$

Clearly the same inequality then holds for $r_{1}, \ldots, r_{m}, s_{1}, \ldots, s_{n}$ rational and then even real by a density argument. This implies that $\Delta(\Phi, \Psi) \leq \sigma$.

Now suppose $0<r<1$. We recall that an $r$-norm on a real or complex vector space $X$ is a map $x \rightarrow\|x\|_{X}$ such that:

(1) $\|x\|_{X}>0$ if $x \neq 0$,

(2) $\|\alpha x\|_{X}=|\alpha|\|x\|_{X}$ for $\alpha \in \mathbb{K}, x \in X$ 
Here $\mathbb{K}=\mathbb{R}$ or $\mathbb{K}=\mathbb{C}$. Notice that in [18] this is called an $r$-subadditive quasinorm. If the metric $d\left(x_{1}, x_{2}\right)=\left\|x_{1}-x_{2}\right\|_{X}^{r}$ makes $X$ complete we say that $X$ is an $r$-normed quasi-Banach space.

Now suppose $X$ and $Y$ are Banach spaces (so that $X$ and $Y$ are also $r$-normed quasi-Banach spaces for any $0<r<1$.) We define $d_{r}(X, Y)$ to be the infimum of $d\left(B_{X}, B_{Y}\right)$ over all linear isometric embeddings of $X$ and $Y$ into an $r$-normed quasi-Banach space $Z$. Note here that the $r$-norm does not induce a metric on $Z$ so this is not covered by the preceding theorem. There is, however an analogue of Theorem 2.3 for this situation. If $(\Phi, \Psi) \in \mathcal{F}_{h}$ then we define $\Delta_{r}(\Phi, \Psi)$ to be the least constant $\Delta_{r}$ such that we have for any $x_{1}, \ldots, x_{m} \in X$ and $y_{1}, \ldots, y_{n} \in Y$ that

$$
\left|\left\|\sum_{i=1}^{m} x_{i}-\sum_{j=1}^{n} \Psi\left(y_{j}\right)\right\|_{X}^{r}-\left\|\sum_{i=1}^{m} \Phi\left(x_{i}\right)-\sum_{j=1}^{n} y_{j}\right\|_{Y}^{r}\right| \leq \Delta_{r}^{r}\left(\sum_{i=1}^{m}\left\|x_{i}\right\|_{X}^{r}+\sum_{j=1}^{n}\left\|y_{j}\right\|_{Y}^{r}\right) .
$$

In an exactly analogous fashion we may prove:

Theorem 2.6. If $X$ and $Y$ are Banach spaces then

$$
d_{r}(X, Y)=\inf _{(\Phi, \Psi) \in \mathcal{F}_{h}} \Delta_{r}(\Psi, \Phi)
$$

\section{COMPARison OF METRICS}

Proposition 3.1. Suppose $X$ and $Y$ are Banach spaces and $(\Phi, \Psi) \in \mathcal{F}_{h}(X, Y)$. Let $\sigma=\Delta(\Phi, \Psi)$. Then

(1) Given $y \in Y$ there exists $x \in X$ with $\|x\|_{X} \leq\|y\|_{Y}$ and $\|y-\Phi(x)\|_{Y} \leq 2 \sigma\|y\|_{Y}$

(2) If $x \in X$ then $\|\Phi(x)\|_{Y} \geq(1-\sigma)\|x\|_{X}$.

(3) If $x_{1}, \ldots, x_{n} \in X$ and $\sum_{k=1}^{n} x_{k}=0$ then

$$
\left\|\sum_{k=1}^{n} \Phi\left(x_{k}\right)\right\|_{Y} \leq \sigma \sum_{k=1}^{n}\left\|x_{k}\right\|_{X}
$$

Proof. (1) Just take $x=\Psi(y)$. Then $\|x-\Psi(y)\|_{X}=0$ so that $\|y-\Phi(x)\|_{Y} \leq$ $\sigma\left(\|x\|_{X}+\|y\|_{Y}\right) \leq 2 \sigma\|y\|_{Y}$. (2) and (3) are immediate from the definition of $\Delta(\Phi, \Psi)$.

In a very similar way we can establish:

Proposition 3.2. Suppose $0<r<1$. Suppose $X$ and $Y$ are Banach spaces and $(\Phi, \Psi) \in \mathcal{F}_{h}(X, Y)$. Let $\sigma=\Delta_{r}(\Phi, \Psi)$. Then

(1.) Given $y \in Y$ there exists $x \in X$ with $\|x\|_{X} \leq\|y\|_{Y}$ and $\|y-\Phi(x)\|_{Y} \leq$ $2^{1 / r} \sigma\|y\|_{Y}$

(2.) If $x \in X$ then $\|\Phi(x)\|_{Y} \geq\left(1-\sigma^{r}\right)^{1 / r}\|x\|_{X}$.

(3.) If $x_{1}, \ldots, x_{n} \in X$ and $\sum_{k=1}^{n} x_{k}=0$ then

$$
\left\|\sum_{k=1}^{n} \Phi\left(x_{k}\right)\right\|_{Y} \leq \sigma\left(\sum_{k=1}^{n}\left\|x_{k}\right\|_{X}^{r}\right)^{1 / r}
$$


Proposition 3.3. Let $X$ and $Y$ be Banach spaces and suppose $\Phi: X \rightarrow Y$ is a homogeneous map satisfying $\frac{1}{2}\|x\|_{X} \leq\|\Phi(x)\|_{Y} \leq\|x\|_{X}$ such that for a constant $0 \leq \sigma<1$ we have:

(1) Given $y \in Y$ there exists $x \in X$ with $\|x\|_{X} \leq\|y\|_{Y}$ and $\|y-\Phi(x)\|_{Y} \leq \sigma\|y\|_{Y}$

(2) If $x_{1}, x_{2}, x_{3} \in X$ and $\sum_{k=1}^{3} x_{k}=0$ then

$$
\left\|\sum_{k=1}^{3} \Phi\left(x_{k}\right)\right\|_{Y} \leq \sigma \sum_{k=1}^{3}\left\|x_{k}\right\|_{X}
$$

Then if $\Psi: Y \rightarrow X$ is a homogenous map satisfying $\|\Psi(y)\|_{X} \leq\|y\|_{Y}$ and $\| y-$ $\Phi(\Psi(y))\left\|_{Y} \leq \sigma\right\| y \|_{Y}$ (whose existence is guaranteed by (1)) we have

$$
\left|\|x-\Psi(y)\|_{X}-\|y-\Phi(x)\|_{Y}\right| \leq 6 \sigma\left(\|x\|_{X}+\|y\|_{Y}\right) .
$$

Furthermore for each $0<r<1$ there is a universal constant $C=C(r)$ such that $\Delta_{r}(\Phi, \Psi) \leq C \sigma$.

If further we have:

(3) If $x_{1}, \ldots, x_{n} \in X$ and $\sum_{k=1}^{n} x_{k}=0$ then

$$
\left\|\sum_{k=1}^{n} \Phi\left(x_{k}\right)\right\|_{Y} \leq \sigma \sum_{k=1}^{n}\left\|x_{k}\right\|_{X}
$$

then $\Delta(\Phi, \Psi) \leq 20 \sigma$.

Proof. Suppose $x \in X$ and $y \in Y$. Then

$$
\|\Phi(x)-\Phi(\Psi(y))-\Phi(x-\Psi(y))\|_{Y} \leq 2 \sigma\left(\|x\|_{X}+\|y\|_{Y}\right) .
$$

Hence

$$
\|\Phi(x)-y-\Phi(x-\Psi(y))\|_{Y} \leq 3 \sigma\left(\|x\|_{X}+\|y\|_{Y}\right) .
$$

It follows that

$$
\|\Phi(x)-y\|_{Y} \leq\|x-\Psi(y)\|_{X}+3 \sigma\left(\|x\|_{X}+\|y\|_{Y}\right)
$$

and

$$
\|x-\Psi(y)\|_{X} \leq\|\Phi(x)-y\|_{Y}+6 \sigma\left(\|x\|_{X}+\|y\|_{Y}\right) .
$$

This proves the first part of the Proposition.

Notice that the same proof yields that for $0<r<1$,

$$
\left|\|x-\Psi(y)\|_{X}^{r}-\|y-\Phi(x)\|_{Y}^{r}\right| \leq 6^{r} \sigma^{r}\left(\|x\|_{X}+\|y\|_{Y}\right)^{r} .
$$

Now suppose $0<r \leq 1$ and that there is a constant $\tau$ so that for any $x_{1}, \ldots, x_{n} \in$ $X$ with $\sum_{i=1}^{n} x_{i}=0$ we have

$$
\left\|\sum^{n} \Phi\left(x_{i}\right)\right\|_{Y} \leq \tau\left(\sum^{n}\left\|x_{i}\right\|_{X}^{r}\right)^{1 / r} .
$$


First suppose $y_{1}, \ldots, y_{n} \in Y$ and $\sum_{i=1}^{n} y_{i}=0$. Let $v=\sum_{i=1}^{n} \Psi\left(y_{i}\right)$. Then

$$
\left\|\sum_{i=1}^{n} \Phi\left(\Psi\left(y_{i}\right)\right)-\Phi(v)\right\|_{Y} \leq 2^{1 / r} \tau\left(\sum_{i=1}^{n}\left\|y_{i}\right\|_{Y}^{r}\right)^{1 / r}
$$

Thus

$$
\left\|\sum_{i=1}^{n} y_{i}-\Phi(v)\right\|_{Y} \leq\left(2^{1 / r} \tau+\sigma\right)\left(\sum_{i=1}^{n}\left\|y_{i}\right\|_{Y}^{r}\right)^{1 / r}
$$

It follows that

$$
\|v\|_{X} \leq 2\|\Phi(v)\|_{Y} \leq\left(2^{1+1 / r} \tau+2 \sigma\right)\left(\sum_{i=1}^{n}\left\|y_{i}\right\|_{Y}^{r}\right)^{1 / r}
$$

Now suppose that $x_{1}, \ldots, x_{m} \in X$ and $y_{1}, \ldots, y_{n} \in Y$. Let $u=\sum_{i=1}^{m} x_{i}$ and $v=\sum_{j=1}^{n} y_{j}$. Then

$$
\left|\|u-\Psi(v)\|_{X}^{r}-\|v-\Phi(u)\|_{Y}^{r}\right| \leq 6^{r} \sigma^{r}\left(\sum_{i=1}^{m}\left\|x_{i}\right\|_{X}^{r}+\sum_{j=1}^{n}\left\|y_{j}\right\|_{Y}^{r}\right) .
$$

However we also have:

$$
\left\|\Psi(v)-\sum_{j=1}^{n} \Psi\left(y_{j}\right)\right\|_{Y}^{r} \leq 2\left(2^{1+1 / r} \tau+2 \sigma\right)^{r} \sum_{j=1}^{n}\left\|y_{j}\right\|_{Y}^{r}
$$

and

$$
\left\|\Phi(u)-\sum_{i=1}^{m} \Phi\left(x_{i}\right)\right\|_{X}^{r} \leq 2 \tau^{r} \sum_{i=1}^{m}\left\|x_{i}\right\|_{X}^{r} .
$$

Combining these we have

$$
\Delta_{r}(\Phi, \Psi) \leq\left(6^{r} \sigma^{r}+2 \tau^{r}+2\left(2^{1+1 / r} \tau+2 \sigma\right)^{r}\right)^{1 / r}
$$

The final part of the Proposition is then immediate taking $r=1$ and $\tau=\sigma$.

For the remaining case we observe that for $0<r<1$ (2) implies that (3.1) holds with a constant $\tau=C \sigma$ where $C$ depends on $r$. This is a well-known calculation first observed in [16] (see also [18] p.91). Suppose $x_{1}, \ldots, x_{n}$ are nonzero in $X$ and $x^{*} \in X^{*}$ with $\left\|x^{*}\right\|_{X^{*}}=1$. Define a linear map $T: \ell_{r}^{n} \rightarrow X$ by $T e_{i}=x_{i} /\left\|x_{i}\right\|_{X}$; then $\|T\| \leq 1$ and (2) implies that the functional $F(\xi)=x^{*}(\Phi T(\xi))$ satisfies

$$
\left|F\left(\xi_{1}+\xi_{2}\right)-F\left(\xi_{1}\right)-F\left(\xi_{2}\right)\right| \leq 2 \sigma\left(\left\|\xi_{1}\right\|_{r}+\left\|\xi_{2}\right\|_{r}\right)
$$

Appealing to Lemma 5.8 of [18] we have

$$
\left|x^{*}\left(\Phi\left(\sum_{i=1}^{n} x_{i}\right)\right)-\sum_{i=1}^{n} x^{*}\left(\Phi\left(x_{i}\right)\right)\right| \leq 2\left(\sum_{i=1}^{n}(2 / i)^{1 / r}\right) \sigma\left(\sum_{i=1}^{n}\left\|x_{i}\right\|_{X}^{r}\right)^{1 / r} .
$$

This establishes the Proposition, since $\sum_{i=1}^{\infty}(2 / i)^{1 / r}<\infty$. 
Lemma 3.4. Suppose $\epsilon>0$. Then there exists $\sigma=\sigma(\epsilon)>0$ so that if $X$ and $Y$ are real Banach spaces and $(\phi, \psi) \in \mathcal{F}\left(B_{X}, B_{Y}\right)$ with $D(\phi, \psi)<\sigma$ then there exists a homogeneous map $\Phi: X \rightarrow Y$ such that $\frac{1}{2}\|x\|_{X} \leq\|\Phi(x)\|_{Y} \leq\|x\|_{X}$ for $x \in X$ and:

1. Given $y \in Y$ there exists $x \in X$ with $\|x\|_{X} \leq\|y\|_{Y}$ and $\|y-\Phi(x)\|_{Y} \leq \epsilon\|y\|_{Y}$

2. If $x_{1}, x_{2}, x_{3} \in X$ and $\sum_{k=1}^{3} x_{k}=0$ then

$$
\left\|\sum_{k=1}^{3} \Phi\left(x_{k}\right)\right\|_{Y} \leq \epsilon\left(\sum_{k=1}^{3}\left\|x_{k}\right\|_{X}\right) .
$$

Proof. Suppose first $D(\phi, \psi)=\sigma$. Let $\phi(0)=y \in B_{Y}$. Then there exists $v \in B_{Y}$ with $\|y-v\|_{Y}=1+\|y\|_{Y}$. Thus $\|\psi(v)\|_{X} \geq 1+\|y\|_{Y}-2 \sigma$. It follows that $\|\phi(0)\|_{Y} \leq$ $2 \sigma$. From this it follows similarly that for any $x \in B_{X}$ we have $\|x\|_{X}-4 \sigma \leq$ $\|\phi(x)\|_{Y} \leq\|x\|_{X}+4 \sigma$.

Now let us assume the conclusion of the Lemma is false.

Indeed if this is so we can find a sequence of pairs of Banach spaces $\left(X_{n}, Y_{n}\right)$ and pairs of functions $\left(\phi_{n}, \psi_{n}\right)$ with $D\left(\phi_{n}, \psi_{n}\right)<\frac{1}{n^{2}}$ but such that for every homogeneous map $\Phi: X_{n} \rightarrow Y_{n}$ with $\frac{1}{2}\|x\|_{X_{n}} \leq\|\Phi(x)\|_{X_{n}} \leq\|x\|_{X_{n}}$ either (1) or (2) fails.

We will in particular define $\Phi_{n}: X \rightarrow Y$ to be a homogenous map such that either $\Phi_{n}(x)=\left(1+\frac{4}{n}\right)^{-1} \phi_{n}(x)$ or $\Phi_{n}(x)=-\left(1+\frac{4}{n}\right)^{-1} \phi_{n}(-x)$ when $\|x\|_{X}=\frac{1}{n}$. As long as $n \geq 20$ we then have $\frac{1}{2}\|x\|_{X_{n}} \leq\left\|\Phi_{n}(x)\right\|_{Y_{n}} \leq\|x\|_{X_{n}}$.

Now let $\mathcal{U}$ be a nonprincipal ultrafilter on $\mathbb{N}$. We form the ultraproducts $X_{\mathcal{U}}=$ $\prod_{\mathcal{U}}\left(X_{n}\right)$ and $Y_{\mathcal{U}}=\prod_{\mathcal{U}}\left(Y_{n}\right)$. thus $X_{\mathcal{U}}$ can be realized as the Hausdorff quotient of the seminormed space $\ell_{\infty}\left(X_{n}\right)$ with the seminorm $\|\mathbf{x}\|_{X_{\mathcal{U}}}=\lim _{n \in \mathcal{U}}\left\|x_{n}\right\|_{X_{n}}$ where $\mathbf{x}=\left(x_{n}\right)$. We refer to [13] or [9] for details about ultraproducts.

Define a map $\Omega: X_{\mathcal{U}} \rightarrow Y_{\mathcal{U}}$ by

$$
\Omega(\mathbf{x})=\left(n \phi_{n}\left(\frac{1}{n} x_{n}\right)\right)_{n=1}^{\infty}
$$

(where we defined $\phi_{n}(x)=0$ if $\|x\|_{X_{n}}>1$.). We may argue that $\Omega$ is well-defined since

$$
\frac{1}{n}\left\|x_{n}-u_{n}\right\|_{X_{n}}-\frac{2}{n^{2}} \leq\left\|\phi_{n}\left(\frac{1}{n} x_{n}\right)-\phi_{n}\left(\frac{1}{n} u_{n}\right)\right\|_{Y_{n}} \leq \frac{1}{n}\left\|x_{n}-u_{n}\right\|_{X_{n}}+\frac{2}{n^{2}}
$$

if $\left\|x_{n}\right\|_{X_{n}},\left\|u_{n}\right\|_{X_{n}} \leq n$. The same inequality implies that $\Omega$ is an isometry.

If $\mathbf{y}=\left(y_{n}\right) \in \ell_{\infty}\left(Y_{n}\right)$ we can define $x_{n}=n \psi_{n}\left(y_{n} / n\right)$ for all but finitely many $n$. Then $\left\|\phi_{n}\left(x_{n} / n\right)-y_{n} / n\right\|_{Y_{n}} \leq \frac{2}{n^{2}}$. It follows that $\Omega$ is onto.

We also note that since $\left\|\phi_{n}(0)\right\|_{Y_{n}} \leq \frac{2}{n^{2}}$ we have $\Omega(0)=0$. It follows from the Mazur-Ulam theorem ([21], [29]) that $\Omega$ is linear.

Now notice that if $\left\|x_{n}\right\|_{X_{n}}=1$ then $\Omega(\mathbf{x})=\left(\Phi_{n}\left(x_{n}\right)\right.$ ) (as elements of the ultraproduct). Since $\Phi_{n}$ is homogeneous this means that for every $\mathbf{x} \in X_{\mathcal{U}}$ we have $\Omega(\mathbf{x})=\left(\Phi_{n}\left(x_{n}\right)\right)$.

Since (1) or (2) fails for every $n \geq 20$ we can assume that for some set $P \in \mathcal{U}$ 
exists $\mathbf{y}=\left(y_{n}\right)$ with $y_{n} \in S_{Y_{n}}$ such that if $n \in P$ then $\left\|y_{n}-\Phi_{n}\left(x_{n}\right)\right\| \geq \epsilon$ whenever $\left\|x_{n}\right\|_{X_{n}} \leq 1$. This contradicts the fact that $\Omega$ is an onto isometry.

Similarly if (2) fails then there exist $\mathbf{x}_{k}=\left(x_{k n}\right)$ for $k=1,2,3$ with $\sum_{k=1}^{3}\left\|x_{k n}\right\|_{X_{n}}=$ $1, \sum_{k=1}^{3} x_{k n}=0$ and $\left\|\sum_{k=1}^{3} \Phi_{n}\left(x_{k n}\right)\right\|_{Y_{n}} \geq \epsilon$ for $n \in P$. This contradicts the linearity of $\Omega$.

It is perhaps worth recording a result implicit in this argument:

Proposition 3.5. Suppose $\left(X_{n}\right)$ and $\left(Y_{n}\right)$ are two sequences of real Banach spaces for which $\lim _{n \rightarrow \infty} d_{G H}\left(X_{n}, Y_{n}\right)=0$. Then for any non-principal ultrafilter $\mathcal{U}$ on $\mathbb{N}$ the ultraproducts $\prod_{\mathcal{U}}\left(X_{n}\right)$ and $\prod_{\mathcal{U}}\left(Y_{n}\right)$ are isometric.

Now we are able to establish that for $0<r<1$ we have equivalence for the metrics $d_{G H}$ and $d_{r}$.

Theorem 3.6. Suppose $0<r<1$. Then there is a function $f=f_{r}:(0,1] \rightarrow(0,1]$ with $\lim _{\epsilon \rightarrow 0} f(\epsilon)=0$ such that for every pair of real Banach spaces $X$ and $Y$ we have

$$
2^{-2 / r+1} d_{G H}(X, Y) \leq d_{r}(X, Y) \leq f\left(d_{G H}(X, Y)\right) .
$$

Remark. It would be interesting to find an explicit function $f$ satisfying the conditions of the theorem.

Proof. In fact the upper estimate follows immediately from Proposition 3.3 and Lemma 3.4.

For the lower estimate we begin by noticing that if $(\Phi, \Psi) \in \mathcal{F}_{h}(X, Y)$ and $\Delta_{r}(X, Y)=\sigma$ then for $x_{1}, \ldots, x_{m} \in X$ and $y_{1}, \ldots, y_{n} \in Y$ we have, by putting $u=\sum_{i=1}^{m} x_{i}-\sum_{j=1}^{n} \Psi\left(y_{j}\right)$,

$$
\left\|\sum_{i=1}^{m} \Phi\left(x_{i}\right)-\sum_{j=1}^{n} y_{j}-\Phi(u)\right\|_{Y} \leq 2^{1 / r} \sigma\left(\sum_{i=1}^{m}\left\|x_{i}\right\|_{X}^{r}+\sum_{j=1}^{n}\left\|y_{j}\right\|_{Y}^{r}\right)^{1 / r}
$$

Since $\|\Phi(u)\|_{Y} \leq\|u\|_{X}$ this implies that

$$
\left\|\sum_{i=1}^{m} \Phi\left(x_{i}\right)-\sum_{j=1}^{n} y_{j}\right\|_{Y} \leq\|u\|_{X}+2^{1 / r} \sigma\left(\sum_{i=1}^{m}\left\|x_{i}\right\|_{X}^{r}+\sum_{j=1}^{n}\left\|y_{j}\right\|_{Y}^{r}\right)^{1 / r}
$$

By using this and the symmetrical calculation with $X, Y$ interchanged we obtain

$$
\left|\left\|\sum_{i=1}^{m} x_{i}-\sum_{j=1}^{n} \Psi\left(y_{j}\right)\right\|_{X}-\left\|\sum_{i=1}^{m} \Phi\left(x_{i}\right)-\sum_{j=1}^{n} y_{j}\right\|_{Y}\right| \leq 2^{1 / r} \sigma\left(\sum_{i=1}^{m}\left\|x_{i}\right\|_{X}^{r}+\sum_{j=1}^{n}\left\|y_{j}\right\|_{Y}^{r}\right)^{1 / r}
$$

If we let $\phi$ and $\psi$ be the restrictions of $\Phi$ and $\Psi$ to the respective unit balls and apply the above inequality for $m+n \leq 2$ we obtain that $D(\phi, \psi) \leq 2^{2 / r-1} \sigma$.

We now recall the definition of $\mathcal{K}$-space from [16] or [18]. We say that a Banach space $X$ is a $\mathcal{K}$-space if there is a constant $\kappa$ (we denote the best such constant by $\kappa(X))$ such that whenever $f: X \rightarrow \mathbb{R}$ is a homogeneous function satisfying 
there is a linear functional $g: X \rightarrow \mathbb{R}$ with $|f(x)-g(x)| \leq \kappa\|x\|_{X}$ for all $x \in X$. It is also natural to consider the notion of a $\mathcal{K}_{0}$-space. We say that a Banach space $X$ is a $\mathcal{K}_{0}$-space if there is a constant $\kappa_{0}$ (we denote the best such constant by $\kappa_{0}(X)$ ) such that whenever $f: X \rightarrow \mathbb{R}$ is a homogeneous function, which is bounded on $B_{X}$ and satisfies

$$
\left|f\left(x_{1}+x_{2}\right)-f\left(x_{1}\right)-f\left(x_{2}\right)\right| \leq\left\|x_{1}\right\|_{X}+\left\|x_{2}\right\|_{X}
$$

there is a linear functional $x^{*} \in X^{*}$ with $\left|f(x)-x^{*}(x)\right| \leq \kappa_{0}\|x\|_{X}$ for all $x \in X$. Clearly $\kappa_{0}(X) \leq \kappa(X)$. If $X$ has the Bounded Approximation Property it may be shown that $X$ is a $\mathcal{K}_{0}$-space if and only if $X$ is a $\mathcal{K}$-space. In general however this equivalence is not known.

It is known that a Banach space $X$ is a $\mathcal{K}$-space if $X$ has nontrivial type $([16])$ or if $X^{*}$ is isomorphic to a subspace of $L_{1}$ (see [19]).

Theorem 3.7. Suppose $0<r<1$. Then there is a constant $C=C(r)$ so that if $X$ is a $\mathcal{K}_{0}$-space then for any Banach space $Y, d_{K}(X, Y) \leq C \kappa_{0}(X) d_{r}(X, Y)$.

In particular if $X$ is a real $\mathcal{K}_{0}$-space then for any sequence of Banach spaces $\left(X_{n}\right)$ we have $\lim _{n \rightarrow \infty} d_{G H}\left(X_{n}, X\right)=0$ implies $\lim _{n \rightarrow \infty} d_{K}\left(X_{n}, X\right)=0$.

Remark. Notice that this theorem applies if $X$ is super-reflexive or if $X$ is isomorphic to $c_{0}$ or $C(K)$ for some compact Hausdorff space. As we note later in the case when $X$ is isomorphic to $c_{0}$ or $\ell_{\infty}$ one can show that $\lim _{n \rightarrow \infty} d_{B M}\left(X_{n}, X\right)=0$ whenever $\lim _{n \rightarrow \infty} d_{G H}\left(X_{n}, X\right)=0$ (again for real spaces only).

Proof. Suppose $(\Phi, \Psi) \in \mathcal{F}_{h}(X, Y)$, and $\sigma=\Delta_{r}(\Phi, \Psi)$. Then for $x_{1}, x_{2} \in X$ we have

$$
\left\|\Phi\left(x_{1}+x_{2}\right)-\Phi\left(x_{1}\right)-\Phi\left(x_{2}\right)\right\|_{Y} \leq 2^{2 / r-1} \sigma\left(\left\|x_{1}\right\|_{X}+\left\|x_{2}\right\|_{X}\right) .
$$

If $\left\|y^{*}\right\|_{Y^{*}}=1$ then there is a linear map $g: X \rightarrow \mathbb{R}$ such that

$$
\left|y^{*}(\Phi(x))-g(x)\right| \leq 2^{2 / r-1} \sigma \kappa_{0}(X)\|x\|_{X}
$$

Now if $\sum_{i=1}^{n} x_{i}=0$ we obtain

$$
\left|\sum_{i=1}^{n} y^{*}\left(\Phi\left(x_{i}\right)\right)\right| \leq 2^{2 / r-1} \sigma \kappa_{0}(X) \sum_{i=1}^{n}\left\|x_{i}\right\|_{X}
$$

This in turn implies that

$$
\left\|\sum_{i=1}^{n} \Phi\left(x_{i}\right)\right\| \leq 2^{2 / r-1} \sigma \kappa_{0}(X) \sum_{i=1}^{n}\left\|x_{i}\right\|_{X} .
$$

The result follows on appealing to Proposition 3.3.

Example. We now show by example that the Kadets and Gromov-Hausdorff distances are not equivalent for general Banach spaces. To see this we show that $\lim _{p \rightarrow 1} d_{G H}\left(\ell_{p}, \ell_{1}\right)=0$. It is known $\left(\operatorname{cf}[22]\right.$ or [24]) that $d_{K}\left(\ell_{1}, \ell_{p}\right)=1$ for all $p>1$.

We consider the Mazur map $\phi: B_{\ell_{p}} \rightarrow B_{\ell_{1}}$ which is defined by 
for $\xi=\left(\xi_{n}\right)$. Let $\psi=\phi^{-1}$ and then we compute $D(\phi, \psi)$. We need only estimate

$$
\left|\|\xi-\eta\|_{\ell_{p}}-\|\phi(\xi)-\phi(\eta)\|_{\ell_{1}}\right|
$$

over all $\xi, \eta \in B_{\ell_{p}}$. Clearly

$$
\left|\|\xi-\eta\|_{\ell_{p}}-\|\xi-\eta\|_{\ell_{p}}^{p}\right| \leq 2^{p}-2
$$

For any $a, b \in \mathbb{R}$ we have the estimate

$$
|| a-\left.b\right|^{p}-\left.|\operatorname{sgn} a| a\right|^{p}-\operatorname{sgn} b|b|^{p}|| \leq\left(2^{p-1}-1\right)\left(|a|^{p}+|b|^{p}\right) .
$$

To see this note that if $s, t \geq 0$ we have

$$
s^{p}+t^{p} \leq(s+t)^{p} \leq 2^{p-1}\left(s^{p}+t^{p}\right)
$$

This implies that if $0 \leq s \leq t$ then

$$
(t-s)^{p} \leq t^{p}-s^{p} \leq(t-s)^{p}+\left(2^{p-1}-1\right)\left(s^{p}+(t-s)^{p}\right) .
$$

The required inequality now follows by considering cases.

Now by summing we obtain

$$
\left|\|\xi-\eta\|_{\ell_{p}}^{p}-\|\phi(\xi)-\phi(\eta)\|_{\ell_{1}}\right| \leq 2^{p}-2
$$

This implies that $D(\phi, \psi) \leq 2\left(2^{p}-2\right)$. Hence $d_{G H}\left(\ell_{1}, \ell_{p}\right) \leq 2\left(2^{p}-2\right) \rightarrow 0$ as $p \rightarrow 1$.

\section{Continuity of Certain maps for the Kadets metric}

In this section, we will establish a number of continuity-type results for the Kadets distance. Thus for example Theorem 4.3 below can be interpreted as saying that the map $X \rightarrow X^{*}$ is continuous for the Kadets distance.

Theorem 4.1. Let $Z$ be a Banach space and let $E, F$ be closed subspaces of $Z$. Then

$$
d_{K}(Z / E, Z / F) \leq 2 \Lambda(E, F)
$$

Remark. A somewhat different version of this result can be found in the unpublished manuscript [14].

Proof. Let $X=Z / E$ and $Y=Z / F$. Suppose $\sigma>\Lambda(E, F)$. Let $q_{X}$ and $q_{Y}$ be the respective quotient maps. If $\theta>1$ we can define a homogeneous map $f_{X}: X \rightarrow Z$ such that $q_{X} f_{X}=I_{X}$ and $\left\|f_{X} x\right\|_{Z} \leq \theta\|x\|_{X}$. We make a similar definition of $f_{Y}$. Finally define $\Phi: X \rightarrow Y$ by $\Phi(x)=\theta^{-1} q_{Y} f_{X}(x)$ and $\Psi: Y \rightarrow X$ by $\Psi(y)=\theta^{-1} q_{X} f_{Y}(y)$.

Suppose $x_{1}, \ldots, x_{m} \in X$ and $y_{1}, \ldots, y_{n} \in Y$. Let $u=\sum_{i=1}^{m} x_{i}+\sum_{j=1}^{n} \Psi\left(y_{j}\right)$. Then $f_{X}(u)-\sum_{i=1}^{m} f_{X}\left(x_{i}\right)-\theta^{-1} \sum_{j=1}^{n} f_{Y}\left(y_{j}\right) \in E$. It follows that there exists $z \in F$ with

$$
\left\|f_{X}(u)-\sum^{m} f_{X}\left(x_{i}\right)-\theta^{-1} \sum^{n} f_{Y}\left(y_{j}\right)-z\right\|_{Z} \leq \sigma\left(\theta\left(\|u\|_{X}+\sum^{m}\left\|x_{i}\right\|_{X}\right)+\sum^{n}\left\|y_{j}\right\|_{Y} \cdot\right)
$$


Since $\|u\|_{X} \leq \sum_{i=1}^{m}\left\|x_{i}\right\|_{X}+\sum_{j=1}^{n}\left\|y_{j}\right\|_{Y}$ this implies that

$$
\left\|\theta \Phi(u)-\theta \sum_{i=1}^{m} \Phi\left(x_{i}\right)-\theta^{-1} \sum_{j=1}^{n} y_{j}\right\|_{Y} \leq 2 \sigma \theta\left(\sum_{i=1}^{m}\left\|x_{i}\right\|_{X}+\sum_{j=1}^{n}\left\|y_{j}\right\|_{Y}\right) .
$$

Note $\|\Phi(u)\|_{Y} \leq\|u\|_{X}$ so that if $v=\sum_{i=1}^{m} \Phi\left(x_{i}\right)+\sum_{j=1}^{n} y_{j}$ we obtain that

$$
\|v\|_{Y} \leq\|u\|_{X}+\left(2 \sigma+\left(1-\theta^{-2}\right)\right)\left(\sum_{i=1}^{m}\left\|x_{i}\right\|_{X}+\sum_{j=1}^{n}\left\|y_{j}\right\|_{Y}\right) .
$$

Combined with the corresponding converse inequality this implies that

$$
\Delta(\Phi, \Psi) \leq 2 \sigma+\left(1-\theta^{-2}\right)
$$

The result now follows.

It is interesting to note that there is a converse to this result.

Theorem 4.2. Suppose $X$ and $Y$ are Banach spaces, and suppose $\sigma>d_{K}(X, Y)$. Then there is a Banach space $Z$ with closed subspaces $E, F$ such that $Z / E$ is isometric to $X, Z / F$ is isometric to $Y$ and $\Lambda(E, F) \leq \sigma$. Furthermore $E$ is isometric to $Y$ and $F$ is isometric to $X$.

Proof. Suppose that $(\Phi, \Psi) \in \mathcal{F}_{h}(X, Y)$ with $\Delta(\Phi, \Psi) \leq \sigma$. We apply the construction of Theorem 2.3. As there, we define $Z$ to be algebraically $X \oplus Y$ and then define the norm by

$$
\|(u, v)\|_{Z}=\inf \left(\left\|x_{0}\right\|_{X}+\left\|y_{0}\right\|_{Y}+\sigma \sum_{i=1}^{m}\left\|x_{i}\right\|_{X}+\sigma \sum_{j=1}^{n}\left\|y_{j}\right\|_{Y}\right)
$$

where the infimum is taken over all $x_{0}, \ldots, x_{m} \in X$ and $y_{0}, \ldots, y_{n} \in Y$ such that $u=x_{0}+\sum_{j=1}^{m} x_{j}+\sum_{j=1}^{n} \Psi\left(y_{j}\right)$ and $v=y_{0}+\sum_{j=1}^{m} \Phi\left(x_{j}\right)+\sum_{j=1}^{n} y_{j}$.

Now we have $\Lambda(X, Y) \leq \sigma$, where $X$ is identified with $X \oplus\{0\}$ and $Y$ with $\{0\} \oplus Y$. Let us compute $Z / Y$ : this is easily seen to be isometric to $X$ since

$$
\inf _{y \in Y}\|(x, y)\|_{Z}=\|(x, \Phi(x))\|_{Z}=\sigma\|x\|_{X}
$$

Similarly $Z / X$ is isometric to $Y$.

Remark. If $X$ and $Y$ are separable then so is $Z$ and there is a quotient map of $\ell_{1}$ onto $Z$. It may then be verified that if $\sigma>d_{K}(X, Y), X$ and $Y$ can be represented as $\ell_{1} / E$ and $\ell_{1} / F$ where $\Lambda(E, F) \leq 2 \sigma$.

Theorem 4.3. Suppose $X$ and $Y$ are Banach spaces. Then $d_{K}\left(X^{*}, Y^{*}\right) \leq 2 d_{K}(X, Y)$.

Remark. The reverse inequality (i.e. $\left.d_{K}(X, Y) \leq c d_{K}\left(X^{*}, Y^{*}\right)\right)$ is not valid. In order to see this consider the case $X^{*}=Y^{*}=l_{1}, X=c_{0}, Y=$ predual of $l_{1}$, non-isomorphic to $c_{0}$. Proposition 5.3 of [24] imply that $d_{K}(X, Y) \geq 1 / 2$. On the 
Proof. Suppose $\sigma>d_{K}(X, Y)$. Then as in Theorem 4.2, there is a Banach space $Z$ and closed subspaces $E, F$ with $\Lambda(E, F) \leq \sigma$ and $Z / E=X, Z / F=Y$. We then have $d_{K}\left(X^{*}, Y^{*}\right) \leq \Lambda\left(E^{\perp}, F^{\perp}\right)$. We now use the fact that $\Lambda\left(E^{\perp}, F^{\perp}\right) \leq 2 \Lambda(E, F)$ which is well-known (cf. Theorem 3.4(d) and 3.13 of [24]); we will provide a direct proof. If $z^{*} \in E^{\perp}$ and $\left\|z^{*}\right\|_{Z^{*}}=1$ then for $f \in F$ we have $\left|z^{*}(f)\right| \leq \sigma\|f\|_{Z}$. By the Hahn-Banach theorem there exists $u^{*} \in Z^{*}$ with $\left\|u^{*}\right\|_{Z^{*}} \leq \sigma$ and $z^{*}-u^{*} \in F^{\perp}$. Then $\left\|z^{*}-u^{*}\right\|_{Z^{*}} \leq 1+\sigma$ and so $d\left(z^{*}, B_{F^{\perp}}\right) \leq 2 \sigma$. It follows by symmetry that $\Lambda\left(E^{\perp}, F^{\perp}\right) \leq 2 \sigma$.

Theorem 4.4. If $X, Y$ are Banach spaces then we have

(1) $d_{K}\left(X^{* *}, Y^{* *}\right) \leq d_{K}(X, Y)$

(2) For any fixed ultrafilter $\mathcal{U}$ on $\mathbb{N} d_{K}\left(X_{\mathcal{U}}, Y_{\mathcal{U}}\right) \leq d_{K}(X, Y)$

(3) $d_{K}\left(X^{* *} / X, Y^{* *} / Y\right) \leq 8 d_{K}(X, Y)$.

Proof. For (1) observe simply that $X$ and $Y$ are embedded into a common Banach space $Z$ then $X^{* *}, Y^{* *}$ can be identified with $X^{\perp \perp}, Y^{\perp \perp}$ in $Z^{* *}$ and $\Lambda\left(X^{\perp \perp}, Y^{\perp \perp}\right) \leq$ $\Lambda(X, Y)$. For (2) we may use a similar argument with $Z_{\mathcal{U}}$. For (3) we use 4.2. If $\sigma>d_{K}(X, Y)$ there is a Banach space $Z$ with closed subspaces $E, F$ with $\Lambda(E, F) \leq \sigma$ and such that $Z / E$ is isometric to $X$ and $Z / F$ is isometric to $Y$. Furthermore from the construction we have $Z=E+F$. Consider $Z^{* *}$; then it is easy to verify that $\Lambda\left(E^{\perp \perp}, F^{\perp \perp}\right) \leq \sigma$. Let $Q_{X}: Z \rightarrow X$ and $Q_{Y}: Z \rightarrow Y$ be the quotient maps. Now suppose $z^{* *} \in Z+F^{\perp \perp}$ with $\left\|z^{* *}\right\|_{Z^{* *}}=1$ and that $\epsilon>0$. Then $Q_{Y}^{* *} z^{* *} \in X \subset X^{* *}$ so that there exists $z \in Z$ with $\|z\|_{Z} \leq(1+\epsilon)$ and $Q_{Y} z=$ $Q_{Y}^{* *} z^{* *}$. Thus $z^{* *}-z \in F^{\perp \perp}$. Now pick $e^{* *} \in E^{\perp \perp}$ with $\left\|z^{* *}-z-e^{* *}\right\|_{Z^{* *}} \leq(2+\epsilon) \sigma$. Thus $d\left(z^{* *}, E^{\perp \perp}+Z\right) \leq 2 \sigma$. This and the similar inequality with $E, F$ reversed leads to the estimate $\Lambda\left(Z+F^{\perp \perp}, E^{\perp \perp}+Z\right) \leq 4 \sigma$. Now note that $Z^{* *} /\left(Z+F^{\perp \perp}\right)$ is isometric to $Y^{* *} / Y$ and $Z^{* *} /\left(Z+E^{\perp \perp}\right)$ is isometric to $X^{* *} / X$. Now Theorem 4.1 gives the result.

We now turn to complex interpolation of Banach spaces. We present an approach which encompasses several such situations.

Let $E$ be a complex Banach space and $\mathcal{U}$ is an open subset of the complex plane $\mathbb{C}$ which is conformally equivalent to the unit disk $\mathcal{D}$. We define an interpolation field $\mathcal{X}$ to be a vector space of $E$-valued analytic functions $f: \mathcal{U} \rightarrow E$ equipped with a norm $\|\cdot\|_{\mathcal{X}}$ such that $\mathcal{X}$ is a Banach space and such that the following two conditions hold:

(1) If $\varphi: \mathcal{U} \rightarrow \mathcal{D}$ is a conformal equivalence then we have $f \in \mathcal{X}$ if and only if $\varphi f \in \mathcal{X}$ and $\|f\|_{\mathcal{X}}=\|\varphi f\|_{\mathcal{X}}$.

(2) Each evaluation $f \rightarrow f(w)$ for $w \in \mathcal{U}$ is bounded from $\mathcal{X}$ to $E$.

If $\mathcal{X}$ is an interpolation field we define for each $w \in \mathcal{U}$ the Banach space $X_{w}$ to be the set of $x \in E$ such that there exists $f \in \mathcal{X}$ with $f(w)=x$ equipped with the quotient norm $\|x\|_{w}=\inf \left\{\|f\|_{\mathcal{X}}: f(w)=x\right\}$.

This definition is easily seen to encompass the standard definitions of complex interpolation spaces in the literature by taking $\mathcal{U}$ to be the open strip $\mathcal{S}=\{w: 0<$ $\Re w<1\}$. It also covers the interpolation method introduced by Coifman, Cwikel, Rochberg, Sagher and Weiss in [7].

Our main result is that the map $w \rightarrow X_{w}$ is continuous for the Kadets metric. To state our result we introduce the pseudo-hyperbolic metric on $\mathcal{U}$. If $\xi, \eta \in \mathcal{U}$ let $h(\xi, \eta)$ be the pseudo-hyperbolic distance on $\mathcal{U}$ defined by $h(\xi, \eta)=|\varphi(\eta)|(=h(\eta, \xi))$ 
Theorem 4.5. Let $\mathcal{U}$ be an open subset of the complex plane which is conformally equivalent to the unit disk, and let $\mathcal{F}$ be an interpolation field on $\mathcal{U}$. Then if $\xi, \eta \in \mathcal{U}$,

$$
d_{K}\left(X_{\xi}, X_{\eta}\right) \leq 2 h(\xi, \eta)
$$

Proof. The argument is very similar to that of Theorem 4.1. Suppose $\theta>1$. We define a homogenous map $\phi_{\xi}: X_{\xi} \rightarrow \mathcal{X}$ with the property that $\phi_{\xi}(x ; \xi)=x$ and $\left\|\phi_{\xi}(x)\right\|_{\mathcal{X}} \leq \theta\|x\|_{\xi}$. Define $\Phi: X_{\xi} \rightarrow X_{\eta}$ by $\Phi(x)=\theta^{-1} \phi_{\xi}(x ; \eta)$ and similarly let $\Psi: X_{\eta} \rightarrow X_{\xi}$ be defined by $\Psi(y)=\theta^{-1} \phi_{\eta}(y ; \xi)$.

Now suppose $x_{1}, \ldots, x_{m} \in X_{\xi}$ and $y_{1}, \ldots, y_{n} \in X_{\eta}$. Let $u=\sum_{i=1}^{m} x_{i}+\sum_{j=1}^{n} \Psi\left(y_{j}\right)$ and $v=\sum_{i=1}^{m} \Phi\left(x_{i}\right)+\sum_{j=1}^{n} y_{j}$. We note that

$$
\phi_{\xi}(u ; \xi)-\sum_{i=1}^{m} \phi_{\xi}\left(x_{i} ; \xi\right)-\theta^{-1} \sum_{j=1}^{n} \phi_{\eta}\left(y_{j} ; \xi\right)=0 .
$$

Let $\varphi$ be a conformal map of $\mathcal{U}$ onto $\mathcal{D}$ with $\varphi(\xi)=0$. Then there exists $f \in \mathcal{X}$ with

$$
\phi_{\xi}(u)-\sum_{i=1}^{m} \phi_{\xi}\left(x_{i}\right)-\theta^{-1} \sum_{j=1}^{n} \phi_{\eta}\left(y_{j}\right)=\varphi f \text {. }
$$

Using the fact that $\|f\|_{\mathcal{X}}=\|\varphi f\|_{\mathcal{X}}$, we obtain

$$
\left\|\theta \Phi(u)-\theta \sum_{i=1}^{m} \Phi\left(x_{i}\right)-\theta^{-1} \sum_{j=1}^{n} y_{j}\right\|_{\eta} \leq 2 \theta|\varphi(\eta)|\left(\sum_{i=1}^{m}\left\|x_{i}\right\|_{\xi}+\sum_{j=1}^{n}\left\|y_{j}\right\|_{\eta}\right) .
$$

It follows that

$$
\|v\|_{\eta} \leq\|u\|_{\xi}+\left(2 h(\xi, \eta)+\left(1-\theta^{-2}\right)\right)\left(\sum_{i=1}^{m}\left\|x_{i}\right\|_{\xi}+\sum_{j=1}^{n}\left\|y_{j}\right\|_{\eta}\right) .
$$

With the symmetrical inequality this leads to

$$
\Delta(\Phi, \Psi) \leq 2 h(\xi, \eta)+\left(1-\theta^{-2}\right)
$$

and the result follows.

We can apply these results to standard interpolation couples. Let $X_{0}, X_{1}$ be a Banach couple and let $X_{\theta}=\left[X_{0}, X_{1}\right]_{\theta}$ be the standard complex interpolation space obtained by the Calderon method.

Corollary 4.6. If $0<\theta<\phi<1$ then

$$
d_{K}\left(X_{\theta}, X_{\phi}\right) \leq 2 \frac{\sin (\pi(\phi-\theta) / 2)}{\sin (\pi(\phi+\theta) / 2)}
$$

Proof. Define

$$
\varphi(z)=\frac{\sin (\pi(z-\theta) / 2)}{\sin (\pi(z+\theta) / 2)}
$$

Then $\varphi$ is a conformal mapping of the strip $\{z: 0<\Re z<1\}$ onto the open unit disk with $\varphi(\theta)=0$. 
Corollary 4.7. If $1<p<q<\infty$ then for the (complex) spaces $\ell_{p}, \ell_{q}$ we have:

$$
d_{K}\left(\ell_{p}, \ell_{q}\right) \leq 2 \frac{\sin (\pi(1 / p-1 / q) / 2)}{\sin (\pi(1 / p+1 / q) / 2)}
$$

Remark. In fact Corollary 4.7 holds for the corresponding real spaces. One way to see this is to note that the pair $(\Phi, \Psi)$ of Theorem 4.4 can be can be constructed even in the case $\theta=1$ and then map real sequences to real sequences.

Remark. The estimate of Corollary 4.6 improves on previous estimates ([15], [22]). The best known lower estimate $([23])$ is

$$
d_{K}\left(\ell_{p}, \ell_{q}\right) \geq 2^{1 / p-1}-2^{1 / q-1} .
$$

Remark. We can also consider complex interpolation of quasi-Banach spaces. If we fix $0<r<1$ and allow $E$ in our definition to be an $r$-Banach space it is easy to show that the map $\xi \rightarrow X_{\xi}$ is continuous for the pseudo-metric $d_{r}$. By interpolating between $\ell_{r}$ when $r<1$ and $\ell_{2}$ one can then see that $\lim _{p \rightarrow 1} d_{G H}\left(\ell_{p}, \ell_{1}\right)=0$ as verified directly in the previous section.

\section{The Kadets and Gromov-HausdorfF topologies}

Let $\aleph$ be any arbitrary but fixed cardinal. Then we may consider the pseudometric space $\mathcal{B}_{\aleph}$ of all Banach spaces with density character at most $\aleph$ with the Kadets or Gromov-Hausdorff pseudo-metrics. We note that there are examples of non-isomorphic Banach spaces for which $d_{K}(X, Y)=0$. An easy way to construct examples is to fix $1<s<\infty$ and take a sequence $1<p_{n}<\infty$ with $\lim _{n \rightarrow \infty} p_{n}=s$, but $p_{n} \neq s$ for all $n$. Then $X=\ell_{s} \oplus_{2} \ell_{2}\left(\ell_{p_{n}}\right)$ and $Y=\ell_{2}\left(\ell_{p_{n}}\right)$ satisfy $d_{K}(X, Y)=0$. This follows easily from the estimate from Corollary 4.6 ,

$$
d_{K}\left(\ell_{2}\left(\ell_{p_{n}}\right), \ell_{2}\left(\ell_{r_{n}}\right)\right) \leq 2 \sup _{n} \frac{\left|\sin \left(\pi\left(p_{n}^{-1}-r_{n}^{-1}\right) / 2\right)\right|}{\sin \left(\pi\left(p_{n}^{-1}+r_{n}^{-1}\right) / 2\right)}
$$

Clearly these spaces are non-isomorphic. If one takes $s=1$ then one gets an example where $d_{G H}(X, Y)=0$ but $d_{K}(X, Y)=1$ since $\ell_{1}$ embeds in $X$ but not $Y$.

We will be interested in this section in the topology of the (pseudo-)metric spaces $\mathcal{B}_{\aleph}$ with the Kadets or Gromov-Hausdorff distances (we will sometimes use the term metric with the understanding that the spaces actually considered are the Hausdorff quotients). We consider a fixed cardinal $\aleph$ to avoid certain set-theoretic problems; the collection of all Banach spaces fails to be a set. The most interesting choice of $\aleph$ is of course $\aleph_{0}$ the set of separable Banach spaces; however in computing duals, biduals etc. it is necessary to consider larger cardinals. We observe that each set $\mathcal{B}_{\aleph}$ is clopen (closed and open) in any larger $\mathcal{B}_{\aleph^{\prime}}$.

Let $\mathcal{P}$ be a property of Banach spaces; then for each cardinal $\aleph$ we may consider the set $\mathcal{P}=\mathcal{P}_{\aleph}$ of all $X \in \mathcal{B}_{\aleph}$ with property $\mathcal{P}$, so that we can think of $\mathcal{P}$ as a set. We will say (following [24]) that $\mathcal{P}$ is stable if there exists a fixed $\alpha>0$ so that $X \in \mathcal{P}$ and $d_{K}(X, Y)<\alpha$ imply $Y \in \mathcal{P}$. We will say that $\mathcal{P}$ is respectively clopen, open, closed if (for each $\aleph$ ) the set $\mathcal{P}$ is respectively clopen, open or closed for the Kadets pseudo-metric. Obviously a stable property is clopen; also the negation of 
There are many known examples of stable properties. Let us list some:

(1) $X$ is separable.

(2) $X$ does not contain $\ell_{1}$.

(3) $X$ is reflexive.

(4) $X$ is super-reflexive.

(5) $X$ has nontrivial type.

See [22], [23] and [24], where other stable properties are also discussed. . We do not know of any examples of clopen properties which are not stable.

The following Proposition is trivial from Theorem 4.3 and Theorem 4.4. Notice that this proposition was known for stable properties (cf. [24], [2]) and for open properties only under some restrictions ([24], [2]).

Proposition 5.1. Suppose $\mathcal{P}$ is a stable (respectively clopen, open, closed) property; then the properties $\mathcal{P}^{*}=\left\{X: X^{*} \in \mathcal{P}\right\}, \mathcal{P}^{c o}=\left\{X: X^{* *} / X \in \mathcal{P}\right\}$ and $\mathcal{P}^{\mathcal{U}}=\left\{X: X_{\mathcal{U}} \in \mathcal{P}\right\}$ for some fixed ultrafilter $\mathcal{U}$ on $\mathbb{N}$ are also stable (respectively, clopen, open, closed).

Note that for (1) and (2) above this leads to new stable properties:

$\left\{X: X^{*}\right.$ is separable $\}$ and $\left\{X: X^{*}\right.$ does not contain $\left.\ell_{1}\right\}$.

Let us also mention some examples of open properties:

(6) $X$ is isomorphic to $\ell_{1}$.

(7) $X$ is isomorphic to $\ell_{\infty}$.

(8) $X$ is injective.

(9) $X$ is isomorphic to $c_{0}$.

We refer again to [22], [23] and [24]. In fact, in each example it is easy to show additionally that the Kadets distance defines a topology equivalent on the set to the Banach-Mazur distance, i.e. $\lim _{n \rightarrow \infty} d_{K}\left(X_{n}, X\right)=0$ if and only if $\lim _{n \rightarrow \infty} d_{B M}\left(X_{n}, X\right)=0$. Notice that we also have that the following are open properties by Proposition 5.1:

(10) $X^{*}$ is isomorphic to $\ell_{1}$.

(11) $X$ is a $\mathcal{L}_{1}$-space.

Let us add to the list the following simple further open properties:

Proposition 5.2. The properties " $X$ is isomorphic to a subspace of $c_{0}$ " and " $X$ is isomorphic to a subspace of $\ell_{\infty}$ " are open.

Proof. We prove only the former assertion. Assume that $X$ is a Banach space with Banach-Mazur distance less than $\lambda$ to a subspace of $c_{0}$. Suppose $d_{K}(X, Y)<\frac{1}{2 \lambda+1}$. Then $Y$ is separable. There is a separable Banach space $Z$ which contains $X$ and $Y$ isometrically so that $\sigma=\Lambda(X, Y)<\frac{1}{2 \lambda+1}$. Let $T: X \rightarrow c_{0}$ be a linear operator with $\|x\|_{X} \leq\|T x\|_{c_{0}} \leq \lambda\|x\|_{X}$. Then $T$ may be extended to an operator $T: Z \rightarrow c_{0}$ with $\|T\| \leq 2 \lambda$ (cf [30]). If $y \in Y$ then there exists $x \in X$ with $\|x\|_{X} \leq\|y\|_{Y}$ and $\|x-y\|_{Z} \leq \sigma\|y\|_{Y}$. Then

$$
\|T y\|_{c_{0}} \geq\|T x\|_{c_{0}}-2 \lambda \sigma\|y\|_{Y} \geq(1-\sigma-2 \lambda \sigma)\|y\|_{Y}
$$

Thus $Y$ is also isomorphic to a subspace of $c_{0}$.

Theorem 5.3. The property " $\kappa_{0}(X)<\infty$ " is open.

Proof. Suppose $X$ is a Banach space with $\kappa_{0}=\kappa_{0}(X)<\infty$. Suppose $d_{K}(X, Y)<\sigma$, 
map $\Omega: X \rightarrow Y$ such that for $x_{1}, \ldots, x_{n} \in X$ we have

$$
\left|\left\|\sum_{k=1}^{n} \Omega\left(x_{k}\right)\right\|_{Y}-\left\|\sum_{k=1}^{n} x_{k}\right\|_{X}\right| \leq 14 \sigma \sum_{k=1}^{n}\left\|x_{k}\right\|_{X} .
$$

Now suppose $f: Y \rightarrow \mathbb{R}$ is a homogeneous map which is bounded on $B_{Y}$ and such that $\left|f\left(y_{1}+y_{2}\right)-f\left(y_{1}\right)-f\left(y_{2}\right)\right| \leq\left\|y_{1}\right\|_{Y}+\left\|y_{2}\right\|_{Y}$. Since $f$ is bounded a weak* compactness argument shows the existence of a best approximation $y^{*} \in Y^{*}$ so that $\sup _{y \in B_{Y}}\left|f(y)-y^{*}(y)\right|=M$ is minimized.

Let $\varphi=f-y^{*}$. Suppose $x_{1}, x_{2} \in X$. Then $\left\|\Omega\left(x_{1}+x_{2}\right)-\Omega\left(x_{1}\right)-\Omega\left(x_{2}\right)\right\|_{Y} \leq$ $28 \sigma\left(\left\|x_{1}\right\|_{X}+\left\|x_{2}\right\|_{X}\right)$. Hence

$$
\left|\varphi\left(\Omega\left(x_{1}+x_{2}\right)\right)-\varphi\left(\Omega\left(x_{1}\right)+\Omega\left(x_{2}\right)\right)\right| \leq(28 \sigma M+2)\left(\left\|x_{1}\right\|_{X}+\left\|x_{2}\right\|_{X}\right) .
$$

Now

$$
\left|\varphi\left(\Omega\left(x_{1}\right)+\Omega\left(x_{2}\right)\right)-\varphi\left(\Omega\left(x_{1}\right)\right)-\varphi\left(\Omega\left(x_{2}\right)\right)\right| \leq\left\|x_{1}\right\|_{X}+\left\|x_{2}\right\|_{X}
$$

so that we conclude that

$$
\left|\varphi\left(\Omega\left(x_{1}+x_{2}\right)\right)-\varphi\left(\Omega\left(x_{1}\right)\right)-\varphi\left(\Omega\left(x_{2}\right)\right)\right| \leq(28 \sigma M+3)\left(\left\|x_{1}\right\|_{X}+\left\|x_{2}\right\|_{X}\right) .
$$

It follows that there exists $x^{*} \in X^{*}$ so that

$$
\left|\varphi(\Omega(x))-x^{*}(x)\right| \leq \kappa_{0}(28 \sigma M+3)\|x\|_{X} .
$$

Now suppose $y_{1}, \ldots, y_{n} \in Y$ and $\sum_{k=1}^{n} y_{k}=0$. Let $x=\sum_{k=1}^{n} \Omega^{-1}\left(y_{k}\right)$. Then $\|x\|_{X} \leq 14 \sigma \sum_{k=1}^{n}\left\|y_{k}\right\|_{Y}$.

Notice that

$$
\begin{aligned}
\left|\sum_{k=1}^{n} \varphi\left(y_{k}\right)-\varphi(\Omega(x))\right| & =\left|\sum_{k=1}^{n} \varphi\left(\Omega \Omega^{-1} y_{k}\right)-\varphi(\Omega(x))-x^{*}\left(\sum_{k=1}^{n} \Omega^{-1}\left(y_{k}\right)-x\right)\right| \\
& \leq \kappa_{0}(3+28 \sigma M)(1+14 \sigma) \sum_{k=1}^{n}\left\|y_{k}\right\|_{Y}
\end{aligned}
$$

Now

$$
|\varphi(\Omega(x))| \leq M\|x\|_{X} \leq 14 \sigma M \sum_{k=1}^{n}\left\|y_{k}\right\|_{Y}
$$

so that we finally obtain

$$
\left|\sum_{k=1}^{n} \varphi\left(y_{k}\right)\right| \leq C \sum_{k=1}^{n}\left\|y_{k}\right\|_{Y}
$$

where

$$
C=\left(14 \sigma M+\kappa_{0}(6+56 \sigma M)\right) .
$$

Now define a sublinear functional on $Y$ by

$$
p(y)=\inf \left(\sum^{n} \varphi\left(y_{k}\right)+C \sum^{n}\left\|y_{k}\right\|_{Y}: \sum^{n} y_{k}=y\right)
$$


Notice that if $\sum_{k=1}^{n} y_{k}=y$ then

$$
\sum_{k=1}^{n} \varphi\left(y_{k}\right)+C \sum_{k=1}^{n}\left\|y_{k}\right\|_{Y} \geq \varphi(y)-C\|y\|_{Y}
$$

so that $p$ is well-defined. Let $h$ be any linear functional on $Y$ so that $h(y) \leq p(y)$ for all $y$. Then $h(y) \leq \varphi(y)+C\|y\|_{Y}$ and by applying to $-y$ we have $h(y) \geq$ $\varphi(y)-C\|y\|_{Y}$. Thus $|h(y)-\varphi(y)| \leq C\|y\|_{Y}$.

Now considering $f-y^{*}-h$ we see that we must have $M \leq C$ and so if $(14+$ $\left.56 \kappa_{0}\right) \sigma<1$ we have

$$
M \leq \frac{6 \kappa_{0}}{1-14 \sigma-56 \sigma \kappa_{0}}
$$

and this gives an estimate for $\kappa_{0}(Y)$.

The notions of stable and open properties are most naturally applied to interpolation scales.

Proposition 5.4. Suppose $\left(X_{0}, X_{1}\right)$ is a Banach couple and that $X_{\theta}=\left[X_{0}, X_{1}\right]_{\theta}$. If $\mathcal{P}$ is a stable property and there exists $0<\theta<1$ so that $X_{\theta}$ has property $\mathcal{P}$ then $X_{\phi}$ has $\mathcal{P}$ for every $0<\phi<1$.

Proof. This is immediate from Corollary 4.6.

Remark. This can be applied to each of the stable properties listed above. This yields a number of results, many of which are certainly known to specialists. For the example the case of reflexivity can be deduced from Calderon's original paper [6]. However we feel this general framework for such a result has some interest.

Proposition 5.5. Suppose $\left(X_{0}, X_{1}\right)$ is a Banach couple and that $X_{\theta}=\left[X_{0}, X_{1}\right]_{\theta}$. If $\mathcal{P}$ is an open property and there exists $0<\theta<1$ so that $X_{\theta}$ has property $\mathcal{P}$ then there exists $\epsilon>0$ so that $X_{\phi}$ has $\mathcal{P}$ for every $\phi$ with $|\phi-\theta|<\epsilon$.

Here we single out three special cases which seem to be new and of some interest:

Proposition 5.6. Suppose $\left(X_{0}, X_{1}\right)$ is a Banach couple and that $X_{\theta}=\left[X_{0}, X_{1}\right]_{\theta}$. Suppose there exists $0<\theta<1$ so that $X_{\theta}$ is isomorphic to $c_{0}$ (respectively isomorphic to a subspace of $c_{0}$, resp. isomorphic to $\ell_{1}$ ) then there exists $\epsilon>0$ so that $X_{\phi}$ is isomorphic to $c_{0}$ (respectively isomorphic to a subspace of $c_{0}$, resp. isomorphic to $\ell_{1}$ ) for every $\phi$ with $|\phi-\theta|<\epsilon$.

Remarks. Of course, Propositions 5.4-5.6 apply to general interpolation fields.

Proposition 5.4 suggests it is of interest to make the following definition. Let $X$ be an arbitrary Banach space. We will say that the (Kadets) component, $\mathcal{C}_{X}$ of $X$ is the intersection of all clopen properties containing $X$. Clearly if $\left(X_{0}, X_{1}\right)$ is a Banach couple then for all $0<\theta, \phi<1$ we have that $X_{\phi}$ is in the component of $X_{\theta}$.

We first state some elementary properties of components.

Proposition 5.7. Let $X$ be an arbitrary Banach space. Then:

(1) $Y \in \mathcal{C}_{X}$ if and only if $\mathcal{C}_{Y}=\mathcal{C}_{X}$.

(2) For every $Y \in \mathcal{C}_{X}$ the density character dens $X=$ dens $Y$ and dens $X^{*}=$ dens 
(3) If $Y \in \mathcal{C}_{X}$ and $Y_{1}$ is isomorphic to $Y$ then $Y_{1} \in \mathcal{C}_{X}$.

(4) If $Y$ is an arbitrary Banach space then for any subspace $E$ we have $Y / E \oplus E \in$ $\mathcal{C}_{X}$ if and only if $Y \in \mathcal{C}_{X}$.

Remark. Note that (3) allows us not to specify any special norm on the spaces in (4).

Proof. (1) is elementary. For (2) note that the sets $\{Y: \operatorname{dens} Y=\operatorname{dens} X\}$ and $\left\{Y\right.$ : dens $\left.Y^{*}=\operatorname{dens} X^{*}\right\}$ are both clopen.

For (3) observe as in Proposition 6.7 of [24] that there is a family of isomorphic copies of $Y, Y_{t}$ for $0 \leq t \leq 1$, say, so that $t \rightarrow Y_{t}$ is continuous for the Kadets distance, with $Y_{0}=Y$.

For (4) we use Lemma 5.9 of [24]. Let $Z=Y \oplus_{1} Y / E$ and let $Q: Y \rightarrow Y / E$ be the quotient map. For any $t \in \mathbb{R}$ with $t \neq 0$ we let $G_{t}=\{(t y, Q y): y \in Y\}$. Then it is easy to show that $\lim _{s \rightarrow t} \Lambda\left(G_{s}, G_{t}\right)=0$ for any $t \in \mathbb{R}$. If $t=0$ we define $G_{0}=$ $E \oplus_{1} Y / E$. Then using Lemma 5.9 of [24] we also have $\lim _{s \rightarrow 0} \Lambda\left(G_{s}, G_{0}\right)=0$. Thus the map $t \rightarrow G_{t}$ is continuous for the Kadets distance. However $G_{t}$ is isomorphic to $Y$ for all $t \neq 0$ while $G_{0}=E \oplus_{1} Y / E$.

Proposition 5.8. We have $X \in \mathcal{C}_{\ell_{1}}$ if and only if $X$ is separable and contains a copy of $\ell_{1}$.

Proof. Since the set of $X$ which is both separable and contains a copy of $\ell_{1}$ is stable inclusion is immediate. Conversely suppose $X$ is any separable Banach space containing a subspace $E$ isomorphic to $\ell_{1}$. Then $X$ is in the same component as $\ell_{1} \oplus_{1} X / E$. Let $F$ be the kernel of a quotient map from $\ell_{1}$ onto $X / E$. Let $G=$ $\ell_{1}(F) \oplus \ell_{1}(X / E) \in \mathcal{C}_{\ell_{1}}$. Since the map $Y \rightarrow Y \oplus_{1} X / E$ is trivially seen to be continuous for the Kadets metric the set $\left\{Y \oplus_{1} X / E ; Y \in \mathcal{C}_{\ell_{1}}\right\}$ is connected and meets $\mathcal{C}_{\ell_{1}}$ since $G \oplus_{1}(X / E)$ is isomorphic to $G$. Thus $\ell_{1} \oplus_{1} X / E \in \mathcal{C}_{\ell_{1}}$ and so $X \in \mathcal{C}_{\ell_{1}}$.

Problem 1. What is the component of $c_{0}$ ? Similar techiques to the above Proposition show that this contains all infinite-dimensional subspaces of $c_{0}$. Proposition 5.7 (4) does not help to give any other examples, since being a subspace of $c_{0}$ is a threespace property $([1])$.

Problem 2. What is the component of $\ell_{2}$ ? This is contained in the stable set of all separable super-reflexive spaces. We do not know if it coincides with this set. This is related to Pisier's notion of $\theta$-Hilbertian spaces ([28]); any space which is $\theta$-Hilbertian for $\theta>0$ belongs to $\mathcal{C}_{\ell_{2}}$.

One can obviously introduce the notion of a Gromov-Hausdorff component in an analogous fashion. Clearly the Gromov-Hausdorff component of $X, \mathcal{G}_{X}$ contains $\mathcal{C}_{X}$. Since the map $p \rightarrow \ell_{p}$ is continuous for the Gromov-Hausdorff distance for $p \in[1, \infty)$ we have that $\mathcal{G}_{\ell_{1}}=\mathcal{G}_{\ell_{2}}$. It seems quite possible that this will correspond with the collection of all separable Banach spaces so we ask:

Problem 3. Is the set of all separable Banach spaces connected for the GromovHausdorff distance? We are unable to decide if $c_{0}$ is in $\mathcal{G}_{\ell_{2}}$ so it would be interesting to identify $\mathcal{G}_{c_{0}}$.

Let us take this opportunity to make a few remarks about the Gromov-Hausdorff 
Proposition 5.9. The following properties are open for the Gromov-Hausdorff distance:

(1) $X$ is isomorphic to $c_{0}$

(2) $X$ is isomorphic to $\ell_{\infty}$,

(3) $\kappa_{0}(X)<\infty$

(4) $X$ has nontrivial type.

(5) $X$ has nontrivial cotype.

(6) $X^{*}$ has nontrivial type.

(7) $X^{*}$ has nontrivial cotype.

Furthermore, on the sets defined by properties (1) and (2) Gromov-Hausdorff distance defines a topology equivalent to the Banach-Mazur distance.

Proof. (1) and (2) follow almost immediately from Theorem 3.7, and the remarks above. In a similar way (3) follows from Theorem 3.7 and Theorem 5.3. Then (4) follows since if $X$ has nontrivial type then $\kappa_{0}(X)<\infty$ again by using Theorem 3.7.

To establish (5),(6) and (7) we use Proposition 3.5. Indeed it is immediate from 3.5 that the property " $\ell_{\infty}$ is finitely representable in $X$ " is closed for the GromovHausdorff distance. Let us establish (6) and (7) by showing that for a fixed Banach space $E$ the condition " $E$ is finitely representable in $X^{*}$ " is closed for the GromovHausdorff distance. Then we may take $E=\ell_{1}$ and $E=\ell_{\infty}$.

To show this last statement it suffices to suppose $E$ finite-dimensional. Then if $X_{n}$ converges to $X$ in Gromov-Hausdorff distance i.e. $d_{G H}\left(X_{n}, X\right) \rightarrow 0$ and $E$ is finitely representable in each $X_{n}^{*}$ it is immediate that $E$ is isometric to a subspace of $\left(\prod_{\mathcal{U}}\left(X_{n}\right)\right)^{*}$. Hence by Theorem $3.5 E$ embeds isometrically into the space $X_{\mathcal{U}}^{*}$. Now $\left(X^{*}\right)_{\mathcal{U}}$ embeds naturally into $X_{\mathcal{U}}^{*}$ as a norming subspace by the identification

$$
\mathbf{x}^{*}(\mathbf{x})=\lim _{n \in \mathcal{U}} x_{n}^{*}\left(x_{n}\right) \text {. }
$$

The space of operators $\mathcal{L}\left(E, X_{\mathcal{U}}^{*}\right)$ is naturally a dual space of $E \otimes_{\pi} X_{\mathcal{U}}$ and $\mathcal{L}\left(E,\left(X^{*}\right) \mathcal{U}\right)$ is norming as a subspace. Hence $J: E \rightarrow X_{\mathcal{U}}^{*}$ is an isometry we can find a net $J_{\alpha}: E \rightarrow\left(X^{*}\right)_{\mathcal{U}}$ so that $J_{\alpha} \rightarrow J$ weak* and $\left\|J_{\alpha}\right\| \leq 1$. By the weak* lowersemicontinuity of the norm in $X_{\mathcal{U}}^{*}$ it follows that for any $\epsilon>0$ there exists $\alpha$ with $(1-\epsilon)\|e\|_{E} \leq\left\|J_{\alpha}\right\|_{\left(X^{*}\right)_{\mathcal{U}}} \leq\|e\|_{E}$ for $e \in E$. Hence $E$ is finitely representable in $\left(X^{*}\right) \mathcal{U}$ and thus also in $X^{*}$.

Remark. It has been conjectured by the first author that $\kappa_{0}(X)<\infty$ might be equivalent to the property that $X^{*}$ has finite cotype.

We conclude the paper by showing that the collection of all separable Banach spaces is not itself separable for either the Gromov-Hausdorff or Kadets distances. More precisely we show:

Theorem 5.10. Suppose $1<p<\infty$ and $p \neq 2$. Then the set of Banach spaces $X$ isomorphic to $\ell_{p}$ is not separable in either Kadets or Gromov-Hausdorff distances.

Proof. First notice that since $\kappa_{0}\left(\ell_{p}\right)<\infty$ the two pseudo-metrics $d_{K}$ and $d_{G H}$ define equivalent topologies on the set $\mathcal{I}_{p}$ of all isomorphic copies of $\ell_{p}$. Secondly since $X \rightarrow X^{*}$ is a homeomorphism on the set of reflexive spaces for the Kadets pseudo-metric it suffices to consider $1<p<2$.

We shall consider spaces $\ell_{p}\left(E_{n}\right)$ where each $E_{n}$ is a finite-dimensional Hilbert space. Each such space is isomorphic to $\ell_{p}$ by an old result of Pełczyński [26]. We will prove the following Lemma: 
Lemma 5.11. There exists $\sigma_{0}>0$ so that if $\left(E_{n}\right)$ and $\left(F_{n}\right)$ are two sequences of finite-dimensional Hilbert spaces and $d_{K}\left(\ell_{p}\left(E_{n}\right), \ell_{p}\left(F_{n}\right)\right)<\sigma_{0}$ then there is a bijection $\pi: \mathbb{N} \rightarrow \mathbb{N}$ so that $\frac{1}{4} \operatorname{dim} E_{n} \leq \operatorname{dim} F_{\pi(n)} \leq 10 \operatorname{dim} E_{n}$.

Proof of the lemma. We will write $X=\ell_{p}\left(E_{n}\right)$ and $Y=\ell_{p}\left(F_{n}\right)$. A typical element of $X$ will be denoted $\mathbf{x}=\left(x_{n}\right)_{n=1}^{\infty}$ with a similar notation for $Y$. We also adopt the convention that $\eta$ will denote a function of $\sigma$ satisfying $\lim _{\sigma \rightarrow 0} \eta(\sigma)=0$ which may vary from occurence to occurence.

First observe that by Theorem 2.4 there exists a homogeneous, norm-preserving bijection $\Omega: X \rightarrow Y$ with the property that if $\mathbf{x}_{1}, \ldots, \mathbf{x}_{n} \in X$ then

$$
\left|\left\|\sum_{k=1}^{n} \Omega \mathbf{x}_{k}\right\|_{Y}-\left\|\sum_{k=1}^{n} \mathbf{x}_{k}\right\|_{X}\right| \leq 14 \sigma \sum_{k=1}^{n}\left\|\mathbf{x}_{k}\right\|_{X} .
$$

Suppose $\mathbf{u}, \mathbf{v} \in S_{X}$ have disjoint supports, i.e. $\left\|u_{n}\right\|_{E_{n}}\left\|v_{n}\right\|_{E_{n}}=0$ for each $n$. Then $\|\mathbf{u} \pm \mathbf{v}\|_{X}=2^{1 / p}$ and hence $\|\Omega(\mathbf{u}) \pm \Omega(\mathbf{v})\|_{Y} \geq 2^{1 / p}-28 \sigma$. Let $\Omega(\mathbf{u})=\mathbf{y}$ and $\Omega(\mathbf{v})=\mathbf{z}$. Then we have

$$
\begin{aligned}
2^{1 / p}-28 \sigma & \leq\left(\frac{1}{2}\|\mathbf{y}+\mathbf{z}\|_{Y}^{2}+\frac{1}{2}\|\mathbf{y}-\mathbf{z}\|_{Y}^{2}\right)^{1 / 2} \\
& =\left(\left(\sum_{k=1}^{\infty} 2^{-p / 2}\left\|y_{k}+z_{k}\right\|_{F_{k}}^{p}\right)^{2 / p}+\left(\sum_{k=1}^{\infty} 2^{-p / 2}\left\|y_{k}-z_{k}\right\|_{F_{k}}^{p}\right)^{2 / p}\right)^{1 / 2} \\
& \leq\left(\sum_{k=1}^{\infty}\left(\frac{1}{2}\left\|y_{k}+z_{k}\right\|_{F_{k}}^{2}+\frac{1}{2}\left\|y_{k}-z_{k}\right\|_{F_{k}}^{2}\right)^{p / 2}\right)^{1 / p} \\
& =\left(\sum_{k=1}^{\infty}\left(\left\|y_{k}\right\|_{F_{k}}^{2}+\left\|z_{k}\right\|_{F_{k}}^{2}\right)^{p / 2}\right)^{1 / p} \\
& \leq\left(\sum_{k=1}^{\infty} \max \left(\left\|y_{k}\right\|_{F_{k}},\left\|z_{k}\right\|_{F_{k}}\right)^{p}\right)^{1 / p-1 / 2}\left(\sum_{k=1}^{\infty}\left(\left\|y_{k}\right\|_{F_{k}}^{p}+\left\|z_{k}\right\|_{F_{k}}^{p}\right)\right)^{1 / 2} .
\end{aligned}
$$

This implies an estimate that

$$
\left(\sum_{k=1}^{\infty} \max \left(\left\|y_{k}\right\|_{F_{k}},\left\|z_{k}\right\|_{F_{k}}\right)^{p}\right)^{1 / p} \geq 2^{1 / p}-\eta(\sigma)
$$

and hence an estimate

$$
\left(\sum_{k=1}^{\infty} \min \left(\left\|y_{k}\right\|_{F_{k}},\left\|z_{k}\right\|_{F_{k}}\right)^{p}\right)^{1 / p} \leq \eta(\sigma)
$$

Now suppose $\mathbf{y} \in S_{Y}$ is supported on exactly one co-ordinate $m$ say. Let $\Omega^{-1}(\mathbf{y})=\mathbf{x}$. Then we can write $\mathbf{x}=\mathbf{u}+\mathbf{v}$ disjointly where $\|\mathbf{u}\|_{X} \geq\|\mathbf{v}\|_{X}$, and $\|\mathbf{u}\|_{X}^{p} \leq\|\mathbf{v}\|_{X}^{p}+\max \left\|x_{n}\right\|_{E_{n}}^{p}$. Let $\mathbf{w}=\Omega(\mathbf{u})$ and $\mathbf{z}=\Omega(\mathbf{v})$. Then since $\|\mathbf{u}\|_{X},\|\mathbf{v}\|_{X} \leq 1$ we obviously have that $\min \left(\left\|w_{m}\right\|_{F_{m}},\left\|z_{m}\right\|_{F_{m}}\right) \leq \eta$. Now 
so that

$$
\left\|y_{m}-w_{m}-z_{m}\right\|_{F_{m}} \leq 42 \sigma
$$

and hence

$$
\left\|w_{m}+z_{m}\right\|_{F_{m}} \geq 1-42 \sigma .
$$

From this we have an estimate $\max \left(\left\|w_{m}\right\|_{F_{m}},\left\|z_{m}\right\|_{F_{m}}\right) \geq 1-\eta$. This in turn means $\|\mathbf{u}\|_{X} \geq 1-\eta$ and hence an estimate $\max \left\|x_{n}\right\|_{F_{n}} \geq 1-\eta$.

It follows that if $\sigma$ is small enough there is a unique $n=n(\mathbf{y})$ so that $\left\|x_{n}\right\|_{F_{n}} \geq$ $1-\eta$.

Now suppose that $\mathbf{y}_{1}$ and $\mathbf{y}_{2}$ are both unit vectors with the same singleton support $m$. If $n\left(\mathbf{y}_{1}\right) \neq n\left(\mathbf{y}_{2}\right)$ we will have $\left\|\Omega^{-1}\left(\mathbf{y}_{1}\right) \pm \Omega^{-1}\left(\mathbf{y}_{2}\right)\right\|_{X} \geq 2^{1 / p}-\eta$ but $\min \left(\left\|\mathbf{y}_{1} \pm \mathbf{y}_{2}\right\|_{Y}\right) \leq 2^{1 / 2}$. Again for $\sigma$ small enough this is a contradiction. Hence we conclude that $n$ is a function only of $m$.

Applying the same technique to $X$ and $Y$ interchanged we obtain two maps $\pi: \mathbb{N} \rightarrow \mathbb{N}$ and $\rho: \mathbb{N} \rightarrow \mathbb{N}$ such that if $\mathbf{y}=\Omega \mathbf{x}$ and both are unit vectors then:

(a) if $\mathbf{x}$ is supported only at $m$ then $\left\|y_{\pi(m)}\right\|_{F_{\pi(m)}} \geq 1-\eta$ and

(b) if $\mathbf{y}$ is supported only at $m$ then $\left\|x_{\rho(m)}\right\|_{E_{\rho(m)}} \geq 1-\eta$.

Now if $\mathbf{x}$ is supported only at $m$ then $\mathbf{y}=\mathbf{z}+\mathbf{w}$ where $\|\mathbf{z}\|_{Y} \geq 1-\eta$ and $\|\mathbf{w}\|_{Y} \leq \eta$ are disjoint and $\mathbf{z}$ is supported only at $\pi(m)$. Thus

$$
\left\|\mathbf{x}-\Omega^{-1}(\mathbf{z})-\Omega^{-1}(\mathbf{w})\right\|_{X} \leq 42 \sigma
$$

from which it follows that for $\sigma$ small enough we must have $\rho(\pi(m))=m$ and similarly $\pi(\rho(m))=m$. Thus $\pi$ is a bijection.

Pick a maximal subset $\left(\xi_{k}\right)_{k \in J}$ of $B_{E_{m}}$ with $\left\|\xi_{k}-\xi_{l}\right\|_{E_{m}}>\frac{1}{2}$. Then $|J| \leq 5^{\operatorname{dim} E_{m}}$. Let $\mathbf{x}_{k}$ be the element of $X$ with zeros everywhere except $\xi_{k}$ in the $m$ th position. Suppose $\zeta \in S_{F_{\pi(m)}}$; let $\mathbf{z}$ be the similarly defined element of $Y$ with exactly one nonzero element $\zeta$. Let $\mathbf{x}=\Omega^{-1} \mathbf{z}$. Then there exists $k \in J$ with $\left\|\mathbf{x}-\mathbf{x}_{k}\right\|_{X} \leq$ $\frac{1}{2}+\eta(\sigma)$. Then

$$
\left\|\mathbf{z}-\Omega\left(\mathbf{x}_{k}\right)-\Omega\left(\mathbf{x}-\mathbf{x}_{k}\right)\right\|_{Y} \leq 42 \sigma
$$

Hence

$$
\left\|\mathbf{z}-\Omega\left(\mathbf{x}_{k}\right)\right\|_{Y} \leq \frac{1}{2}+\eta(\sigma) .
$$

Now let $\left(\psi_{k}\right)$ be the $\pi(m)$-co-ordinate of $\Omega\left(\mathbf{x}_{k}\right)$. We have

$$
\left\|\zeta-\psi_{k}\right\|_{F_{\pi(m)}} \leq \frac{1}{2}+\eta(\sigma)
$$

For $\sigma$ small enough this implies that $\left(\psi_{k}\right)_{k \in J}$ is a $3 / 4$-net in $B_{F_{\pi(m)}}$ so that

$$
|J| \geq(4 / 3)^{\operatorname{dim} F_{\pi(m)}} .
$$

We conclude that $\operatorname{dim} F_{\pi(m)} \leq C \operatorname{dim} E_{m}$ where $C=\log 5 / \log (4 / 3) \leq 10$. This proves the lemma.

Proof of the theorem. For each infinite subset $M$ of $\mathbb{N}$ form the space $X_{M}=$ $\ell\left(\ell 10^{2 n}\right)$ 


\section{REFERENCES}

1. D. Alspach, Quotients of $c_{0}$ are almost isometric to subspaces of $c_{0}$, Proc. Amer. Math. Soc. 76 (1979), 285-288.

2. J. Alvarez, T. Alvarez and M. Gonzalez, The gap between subspaces and perturbation of non semi-Fredholm operators, Bull. Austral. Math. Soc. 45 (1992), no. 3, 369-376.

3. J. Bourgain, Real isomorphic complex Banach spaces need not be complex isomorphic, Proc. Amer. Math. Soc. 96 (1986), 221-226.

4. R.D. Bourgin, Approximate isometries and finite-dimensional Banach spaces, Trans. Amer. Math. Soc. 207 (1975), 309-328.

5. A.L. Brown, On the space of subspaces of a Banach space, J. London Math. Soc. 5 (1972), no. $1,67-73$.

6. A.P. Calderon, Intermediate spaces and interpolation, the complex method, Studia Math. 24 (1964), 113-190.

7. R.R. Coifman, M. Cwikel, R. Rochberg, Y. Sagher and G. Weiss, A theory of complex interpolation for families of Banach spaces, Adv. Math. 43 (1982), 203-229.

8. M. Daher, Homéomorphismes uniformes entre les sphères unité des espaces d'interpolation, Can. Math. Bull. 38 (1995), 286-294.

9. J. Diestel, H. Jarchow and A. Tonge, Absolutely summing operators, Cambridge University Press, 1995.

10. A. Douady, Le probleme des modules pour les sous-espaces d'un espace analytique donne, Ann. Inst. Fourier 15 (1966), no. 1, 1-94.

11. M. Gromov, Groups of polynomial growth and expanding maps, Inst. Hautes Etudes Sci. Publ. Math. 53 (1981), 183-215.

12. P.M. Gruber, Stability of isometries, Trans. Amer. Math. Soc. 245 (1978), 263-277.

13. S. Heinrich, Ultraproducts in Banach space theory, J. Reine und Angew. Math. 313 (1980), 72-104.

14. R. Janz, Perturbation of Banach spaces, preprint, Konstanz (1987).

15. M.I. Kadets, Note on the gap between subspaces, Funkts. Anal. Prilozhen. 9 (1975), no. 2, 73-74 (Russian); English transl. in Funct. Anal. Appl. 9 (1975), 156-157.

16. N.J. Kalton, The three space problem for locally bounded F-spaces, Compositio Math. 37 (1978), 243-276.

17. An elementary example of a Banach space not isomorphic to its complex conjugate, Can. Math. Bull. 38 (1995), 218-222.

18. N.J. Kalton, N.T. Peck and J.W. Roberts, An F-space sampler, Cambridge University Press, 1984.

19. N.J. Kalton and J.W. Roberts, Uniformly exhaustive submeasures and nearly additive set functions, Trans. Amer. Math. Soc. 278 (1983), 803-816.

20. M.G. Krein, M.A. Krasnoselskii and D.P. Milman, On the defect numbers of linear operators in a Banach space and on some geometric questions, Sbornik Trudov Inst. Matem. AN Ukrainian SSR 11 (1948), 97-112. (Russian)

21. S. Mazur and S. Ulam, Sur les transformations isométriques d'espaces vectoriels normés, C.r. Acad. Sci. Paris 194 (1932), 946-948.

22. M.I. Ostrovskii, On the properties of the opening and related closeness characterizations of Banach spaces, Teor. Funktsii, Funktsional. Anal. i Prilozhen. 42 (1984), 97-107 (Russian); English transl. in Amer. Math. Soc. Transl. (2) 136 (1987), 109-119.

23. _ Banach-Saks properties, injectivity and gaps between subspaces of a Banach space, Teor. Funktsii, Funktsional. Anal. i Prilozhen. 44 (1985), 69-78 (Russian); English transl. in J. Soviet Math. 48 (1990), no. 3, 299-306.

24. - Topologies on the set of all subspaces of a Banach space and related questions of Banach space geometry, Quaestiones Math. 17 (1994), 259-319.

25. Classes of Banach spaces stable and unstable with respect to the opening, Quaestiones Math. 19 (1996), 191-210.

26. A. Pełczyński, Projections in certain Banach spaces, Studia Math. 19 (1960), 209-228.

27. P. Petersen, Gromov-Hausdorff convergence of metric spaces, Differential Geometry: Riemannian Geometry, Edited by R.Greene and S.T.Yau, AMS, Providence, 1993, pp. $489-504$.

28. G. Pisier, Some applications of the complex interpolation method to Banach lattices, J. d'Analyse Math. 35 (1979), 264-281. 
30. A. Sobczyk, Projection of the space $m$ onto its subspace $c_{0}$, Bull. Amer. Math. Soc. 47 (1941), 938-947.

31. S. Szarek, On the existence and uniqueness of complex structure and spaces with "few" operators, Trans. Amer. Math. Soc. 293 (1986), 339-353.

Department of Mathematics, University of Missouri-Columbia, Columbia, MisSOURI 65211, USA

E-mail address: nigel@math.missouri.edu

Mathematical Division, Institute for Low Temperature Physics, 47 Lenin avenue, 310164 KHARKOV, UKRAINE

E-mail address: mostrovskii@ilt.kharkov.ua 